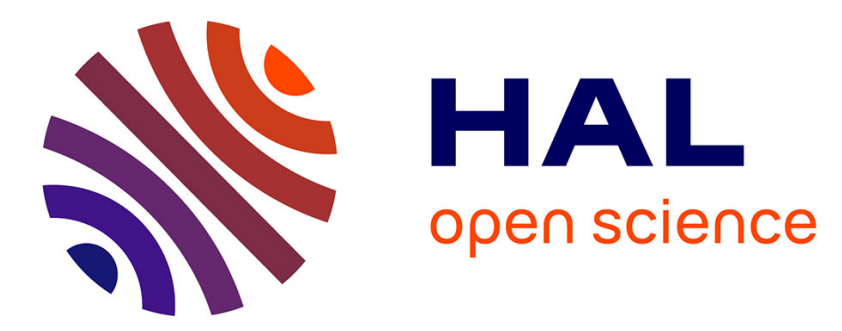

\title{
Structure analysis of direct sampling method in 3D electromagnetic inverse problem: near- and far-field configuration
}

Sangwoo Kang, Marc Lambert

\section{- To cite this version:}

Sangwoo Kang, Marc Lambert. Structure analysis of direct sampling method in 3D electromagnetic inverse problem: near- and far-field configuration. Inverse Problems, 2021, 37 (7), pp.075002. 10.1088/1361-6420/abfe4e . hal-03227222

\section{HAL Id: hal-03227222}

https://hal-centralesupelec.archives-ouvertes.fr/hal-03227222

Submitted on 6 Sep 2021

HAL is a multi-disciplinary open access archive for the deposit and dissemination of scientific research documents, whether they are published or not. The documents may come from teaching and research institutions in France or abroad, or from public or private research centers.
L'archive ouverte pluridisciplinaire HAL, est destinée au dépôt et à la diffusion de documents scientifiques de niveau recherche, publiés ou non, émanant des établissements d'enseignement et de recherche français ou étrangers, des laboratoires publics ou privés. 


\title{
Structure analysis of direct sampling method in three-dimensional inverse electromagnetic scattering problem and its extension to far-field configuration
}

\author{
Sangwoo Kang and Marc Lambert \\ Université Paris-Saclay, CentraleSupélec, CNRS, Laboratoire de Génie Electrique et Electronique de Paris, \\ 91192, Gif-sur-Yvette, France. \\ Sorbonne Université, CNRS, Laboratoire de Génie Electrique et Electronique de Paris, 75252, Paris, France
}

Submitted version, final version@ @ttps://doi.org/10.1088/1361-6420/abfe4e

\begin{abstract}
In this paper, we study the structure analysis of the Direct Sampling Method (DSM) in the threedimensional inverse electromagnetic scattering problem. Even though the DSM is well-known to the robust, fast, and efficient non-iterative type algorithm to estimate the support and shape of unknown inhomogeneities from scattered data, the inhomogeneities might not be detected in the DSM with improper test polarization. To explain the reason for the phenomenon, we carefully analyze the indicator function of DSM using the asymptotic formula of the scattered field under a small volume hypothesis of well-separated inhomogeneities. The analytic representation formula of the indicator function of DSM is presented by establishing the relationship of spherical Bessel functions, the polarization of the incident wave and the test dipole, and the polarization tensor of the inhomogeneities, which depends on their sizes, permittivity, etc. Through such theoretical results, we also show the validity of the guideline to choose the test polarization given by other work. Furthermore, we propose our method to select the polarization of the test dipole for better efficiency. With a similar path of derivation, we also introduce and analyze the indicator function of DSM in far-field configurations. Various numerical simulations with synthetic and experimental data validate our theoretical results and proposals.
\end{abstract}

Keywords: 3D inverse electromagnetic scattering problem, Spherical Bessel function, Structure analysis, Direct sampling method, Orthogonality sampling method

\section{Contents}

1 Introduction 2

2 Three-dimensional direct electromagnetic scattering problem 3

3 Direct sampling method in near-field case 5

3.1 Introduction of direct sampling method and its structure analysis . . . . . . . . . . . . 5

3.2 Numerical simulations . . . . . . . . . . . . . . . . . . . . . . . . . . . . . 9

4 Direct sampling method in far-field case 12

4.1 Introduction of direct sampling method and its structure analysis . . . . . . . . . . . 12

4.2 Numerical simulations with far-field data . . . . . . . . . . . . . . . . 17

5 Illustration with experimental far-field data $\quad 18$

6 Conclusion 21 


\section{Introduction}

The development and the analysis of efficient methods and techniques for solving an inverse scattering problem are of great interest due to their potentials in various applications such as, and without exhaustivity, non-destructive testing $[1,2,3]$, biomedical imaging $[4,5,6]$, and structural imaging $[7,8,9]$ among others. Unfortunately, solving such an inverse problem is known to be difficult because of its inherent illposedness and non-linearity. However, various numerical techniques and algorithms have been developed to deal with such a problem.

Non-iterative type techniques belong to one of the main family of methods because of their advantages such as a low computational cost, no a priori information about the inhomogeneities, and the simplicity of implementation, etc. Among them, we can cite MUltiple SIgnal Classification (MUSIC), Linear Sampling Method (LSM), and migration-type method (Kirchhoff migration, subspace migration, or reverse-time migration), etc., which have been developed and applied in various conditions. These methods are known to provide good results to reconstruct the shapes and locations of unknown inhomogeneities when using enough incident fields [10, 11, 12, 13], but might fail when not as exemplified in [12]. Practically speaking, retrieving the information about the inhomogeneities with only a few number of incident fields is an important issue for various applications such as Synthetic Aperture Radar (SAR) imaging [14, 15, 16, 17] and Ground Penetrating Radar (GPR) [18, 19, 20], etc.

The Direct Sampling Method (DSM) was first developed in [21] for solving the two-dimensional inverse acoustic scattering problems with only a few (one or two) incident fields for imaging and its robustness and stability are shown in $[22,23,24,25]$. Note that the DSM is equivalent to the normalized version of Orthogonality Sampling Method (OSM) proved in [26]. So, we refer to the the relevant works [27, 28, 29]. For the sake of clarity, we denote both the DSM and OSM as DSM. Recently, we have investigated that the structure of the DSM indicator function and validated the correlation with Kirchhoff migration, which is one of the traditional techniques in multi-static configuration [30] and mono-static configuration [31] under the small volume assumption of the well-separated inhomogeneities.

The DSM has been extended to solve two- and three-dimensional inverse electromagnetic scattering problems in [32] with the same conclusions: The DSM is a fast, robust, and efficient technique to reconstruct the supports of the inhomogeneities with only a few incident fields. But, contrary to the acoustic problem, the inhomogeneities might not be detected via DSM if improper polarization of test dipole is chosen. In other words, the main difference between scalar (acoustic case) and vector (electromagnetic case) DSM is the choice of the polarization of the test function. In fact, in the 2D scalar case, the polarization of the test function is nothing but the same as the one of the configuration. Whereas, due to the vectorial aspect of the data in the $3 \mathrm{D}$ electromagnetic case, the choice of test polarization is a crucial parameter of the success of the imaging algorithm. Even though the authors in [32] exhibited the feasibility of DSM, it was not enough to elucidate the relation of polarization of test dipole and the result of DSM in the 3D vectorial case.

It is why, in the following, the analytical formulation of the DSM indicator will be established within the framework of the asymptotic formula of the scattered field for small and well-separated inhomogeneities in near- and far-field configuration. This analytical solution is expressed as a function of the spherical Bessel function of integer order and polarization tensor of the targets, which carries their physical characteristics (e.g., location, size, shape, and permittivity, etc.). Thanks to our analytical analysis, the flowing matters are investigated and explained: (i) the reasons for which an inhomogeneity with a smaller size and/or permittivity among others is difficult to be identified (this phenomenon can arise in the $2 \mathrm{D}$ and $3 \mathrm{D}$ case with acoustic and electromagnetic waves); (ii) the reasons for which some inhomogeneities might not be detected if the polarization of the test dipole is not properly chosen; (iii) the theoretical validation of choosing polarization proposed in [32]. By adopting the idea in [33], which dealt with the improvement of the accuracy of the MUSIC imaging algorithm via a proper selection of the polarization test vector, the direct sampling method with proper test dipole (DSMP) is proposed to handle the second issue in near-field and far-field configurations.

The article is structured as follows. The asymptotic formulations of the direct electromagnetic scattering problem under the near-field and the far-field hypothesis and small volume hypothesis of inhomogeneities are briefly presented in $\S 2$. The structure analysis of the direct sampling method and the corresponding numerical simulations in the near-field and the far-field configuration are presented in $\S 3$ and $\S 4$, respectively. Additional numerical simulations using Fresnel experimental data are presented in $\S 5$. Conclusions and perspectives are drawn in $\S 6$. 


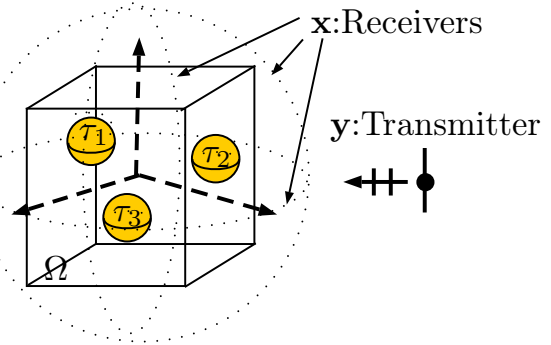

(a) Scattering problem

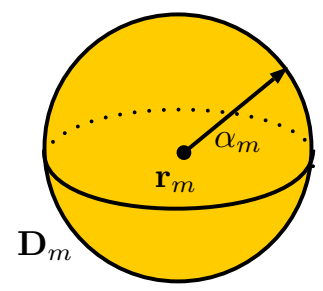

(b) Inhomogeneities $\tau_{m}$

Figure 1: Scattering problem configuration for $M=3$ (left) and sketch of the inhomogeneities $\tau_{m}$ (right).

\section{Three-dimensional direct electromagnetic scattering problem}

In this section, the asymptotic formulation of the three-dimensional direct electromagnetic scattering from the well-separated inhomogeneities under the small volume hypothesis is briefly depicted based on the well-known work [34] (See Figure 1(a) for the sketch), where each inhomogeneity $\tau_{m}$ is represented by

$$
\tau_{m}=\mathbf{r}_{m}+\alpha_{m} \mathbf{D}_{m} \in \Omega, m=1,2, \cdots, M .
$$

Here, $\mathbf{r}_{m}$ and $\alpha_{m}$ are the its location and size, respectively, and $\mathbf{D}_{m}$ characterizes the shape of $\tau_{m}$. For simplicity, we assume that all $\tau_{m}$ are the disks with radius $\alpha_{m}$ (See Figure 1(b)). The collection of all inhomogeneities is denoted by $\tau$, i.e., $\tau=\bigcup_{m} \tau_{m}$. Let us assume that each inhomogeneities is well separated, i.e., there exist positive constant $d_{0}$ such that

$$
0<d_{0} \leq\left|\mathbf{r}_{m}-\mathbf{r}_{m^{\prime}}\right| \text { and } \tau_{m} \cap \tau_{m^{\prime}}, \quad m \neq m^{\prime} .
$$

For the shake of simplicity, all the materials are supposed to be non-magnetic with $\mu_{0}$ as permeability. Let $\varepsilon_{m}$ and $\varepsilon_{0}$ be the electric permittivity of $\tau_{m}$ and of the background, respectively. The angular frequency $\omega_{0}$ is defined as $\omega_{0}=2 \pi f_{0}$, where $f_{0}$ is the frequency in $\mathrm{Hz}$-from now on the time dependence $\mathrm{e}^{-\mathrm{i} \omega_{0} t}$ is omitted. So the piecewise constant electric permittivity $\varepsilon(\mathbf{x})$ and wavenumber $k(\mathbf{x})$ can be defined by

$$
\varepsilon(\mathbf{x})=\left\{\begin{array}{ll}
\varepsilon_{0}, & \text { if } \mathbf{x} \in \mathbb{R}^{3} \backslash \tau \\
\varepsilon_{m}, & \text { if } \mathbf{x} \in \tau
\end{array} \quad \text { and } \quad k(\mathbf{x})= \begin{cases}k_{0}=\omega_{0} \sqrt{\varepsilon_{0} \mu_{0}}, & \text { if } \mathbf{x} \in \mathbb{R}^{3} \backslash \tau \\
k_{m}=\omega_{0} \sqrt{\varepsilon_{m} \mu_{0}}, & \text { if } \mathbf{x} \in \tau,\end{cases}\right.
$$

respectively. Note that the wavelength $\lambda_{0}$ being defined as $\lambda_{0}=2 \pi / k_{0}$.

The total electric fields $\mathbf{E}(\mathbf{x}, \mathbf{y})$ at $\mathbf{x}$ due to a source placed at $\mathbf{y}$ is the solution of following timeharmonic Maxwell's equations:

$$
\left\{\begin{array}{l}
\nabla \times \nabla \times \mathbf{E}(\mathbf{x}, \mathbf{y})-k^{2}(\mathbf{x}) \mathbf{E}(\mathbf{x}, \mathbf{y})=\mathrm{i} \omega_{0} \mu_{0} \mathbf{J} \\
\nu \cdot(\varepsilon(\mathbf{x}) \mathbf{E}(\mathbf{x}, \mathbf{y}))^{+}-\nu \cdot(\varepsilon(\mathbf{x}) \mathbf{E}(\mathbf{x}, \mathbf{y}))^{-}=0 \quad \text { on } \partial \tau
\end{array}\right.
$$

where $\nu$ is outward unit normal to $\tau_{m}$ and $\mathbf{J}$ is induced current of the source. Here, the scripts + and - indicate the limiting direction from outside $\tau_{m}$ and from inside $\tau_{m}$ to boundary of $\tau_{m}$, respectively. The total electric field can be expressed as a sum of incident and scattered electric fields, i.e., $\mathbf{E}(\mathbf{x}, \mathbf{y})=$ $\mathbf{E}^{i}(\mathbf{x}, \mathbf{y})+\mathbf{E}^{s}(\mathbf{x}, \mathbf{y})$.

The incident electric field $\mathbf{E}^{i}(\mathbf{x}, \mathbf{y})$ due to an electric dipole polarized along $\hat{\mathbf{p}}^{t} \in \mathbb{S}^{2}$ and located at $\mathbf{y} \in \Gamma_{\text {inc }}$ is given by

$$
\mathbf{E}^{i}(\mathbf{x}, \mathbf{y})=\mathrm{i} \omega_{0} \mu_{0} I l \underline{\mathbf{G}}(\mathbf{x}, \mathbf{y}) \cdot \hat{\mathbf{p}}^{t},
$$

where $I l$ is the current moment. Here, $\underline{\mathbf{G}}(\mathbf{x}, \mathbf{y})$ is the dyadic Green function which is the solution of

$$
\nabla \times \nabla \times \underline{\mathbf{G}}(\mathbf{x}, \mathbf{y})-k_{0}^{2} \underline{\mathbf{G}}(\mathbf{x}, \mathbf{y})=\delta(\mathbf{x}-\mathbf{y}) \mathbf{I}_{\mathbf{3}},
$$

and it can be expressed as a function of

$$
\underline{\mathbf{G}}(\mathbf{x}, \mathbf{y}):=\left(\underline{\mathbf{I}}_{3}+\frac{1}{k_{0}^{2}} \nabla \nabla\right) g(\mathbf{x}, \mathbf{y})
$$


where $\underline{\mathbf{I}}_{3}$ is $3 \times 3$ identity matrix and $g(\mathbf{x}, \mathbf{y})$ is the Green function of three-dimensional Helmholtz equation, i.e.,

$$
g(\mathbf{x}, \mathbf{y})=\frac{\mathrm{e}^{\mathrm{i} k_{0}|\mathbf{x}-\mathbf{y}|}}{4 \pi|\mathbf{x}-\mathbf{y}|} .
$$

To guarantee for uniqueness of solution, the scattered electric field satisfies the following Silver-Müller radiation condition:

$$
\lim _{|\mathbf{x}| \rightarrow \infty}|\mathbf{x}|\left[\nabla \times \mathbf{E}^{s}(\mathbf{x}, \mathbf{y}) \times \hat{\mathbf{x}}-\mathrm{i} k_{0} \mathbf{E}^{s}(\mathbf{x}, \mathbf{y})\right]=0
$$

uniformly in all directions $\hat{\mathbf{x}}=\mathbf{x} /|\mathbf{x}| \in \mathbb{S}^{2}$.

Following [34], the combining the small volume assumption $\left(\alpha_{m} \sqrt{\varepsilon_{m} / \varepsilon_{0}} \ll \lambda_{0} / 2\right)$ with the noninteraction hypothesis between the inhomogeneities (2), the scattered electric field, which is measured at $\mathbf{x}$ located on the simply connected smooth surface $\Gamma_{\text {obs }}$, has the following asymptotic expansion formula:

Lemma 2.1 (Asymptotic formula of scattered field). Assume that all inhomogeneities are dielectric and sufficiently small $\left(\alpha_{m} \sqrt{\varepsilon_{m} / \varepsilon_{0}} \ll \lambda_{0} / 2\right)$. Then the electric scattered field $\mathbf{E}^{s}(\mathbf{x}, \mathbf{y})$ has the following asymptotic expansion formula for a generic incident field $\mathbf{E}^{i}\left(\mathbf{r}_{m}, \mathbf{y}\right)$

$$
\mathbf{E}^{s}(\mathbf{x}, \mathbf{y})=\omega_{0}^{2} \mu_{0} \sum_{m=1}^{M}\left[\alpha_{m}^{3}\left(\varepsilon_{m}-\varepsilon_{0}\right) \underline{\mathbf{G}}\left(\mathbf{x}, \mathbf{r}_{m}\right) \underline{\mathbf{M}}\left(\frac{\varepsilon_{m}}{\varepsilon_{0}} ; \mathbf{D}_{m}\right) \mathbf{E}^{i}\left(\mathbf{r}_{m}, \mathbf{y}\right)\right]+O\left(\alpha^{4}\right)
$$

where $\alpha:=\max \left\{\alpha_{m}, m=1,2 \cdots, M\right\}$ and $\underline{\mathbf{M}}\left(\varepsilon_{m} / \varepsilon_{0} ; \mathbf{D}_{m}\right) \in \mathbb{C}^{3 \times 3}$ is a polarization tensor related to $\mathbf{D}_{m}$. By combining the form of electric pole given by (5) and asymptotic expansion formula in (10), the electric scattered field $\mathbf{E}^{s}(\mathbf{x}, \mathbf{y})$ in the case of a incident field due to a dipole with a polarization direction $\hat{\mathbf{p}}^{t}$ placed at $\mathbf{y}$ is given by

$$
\mathbf{E}^{s}(\mathbf{x}, \mathbf{y})=\mathrm{i} \omega_{0} \mu_{0} k_{0}^{2} I l \sum_{m=1}^{M}\left[\alpha_{m}^{3}\left(\frac{\varepsilon_{m}-\varepsilon_{0}}{\varepsilon_{0}}\right) \underline{\mathbf{G}}\left(\mathbf{x}, \mathbf{r}_{m}\right) \underline{\mathbf{M}}\left(\frac{\varepsilon_{m}}{\varepsilon_{0}} ; \mathbf{D}_{m}\right) \underline{\mathbf{G}}\left(\mathbf{r}_{m}, \mathbf{y}\right) \cdot \hat{\mathbf{p}}^{t}\right]+O\left(\alpha^{4}\right)
$$

In $[34,35]$ the explicit form of $\underline{\mathbf{M}}\left(\varepsilon_{m} / \varepsilon_{0} ; \mathbf{D}_{m}\right)$ for various forms such as sphere, thin tube, or sheet, is provided. For shake of simplicity, we assume that all inhomogeneities are spherical, so $\underline{\mathbf{M}}\left(\varepsilon_{m} / \varepsilon_{0} ; \mathbf{D}_{m}\right)$ has then the following form:

$$
\underline{\mathbf{M}}\left(\frac{\varepsilon_{m}}{\varepsilon_{0}} ; \mathbf{D}_{m}\right)=\frac{3 \varepsilon_{0}}{2 \varepsilon_{0}+\varepsilon_{m}}\left|\mathbf{D}_{m}\right| \underline{\mathbf{I}}_{3},
$$

and the remainder of either (10) or (11) becomes $O\left(\alpha^{5}\right)$ as shown in [36]. So the field scattered by $M$ small spheres illuminated by a single dipole under the asymptotic hypothesis in near field is given by

$$
\mathbf{E}^{s}(\mathbf{x}, \mathbf{y})=i \omega_{0} \mu_{0} k_{0}^{2} I l \sum_{m=1}^{M} A_{m} \underline{\mathbf{G}}\left(\mathbf{x}, \mathbf{r}_{m}\right) \underline{\mathbf{G}}\left(\mathbf{y}, \mathbf{r}_{m}\right) \hat{\mathbf{p}}^{t}+O\left(\alpha^{5}\right) \text { where } A_{m}=\alpha_{m}^{3} \frac{3\left(\varepsilon_{m}-\varepsilon_{0}\right)}{2 \varepsilon_{0}+\varepsilon_{m}}\left|\mathbf{D}_{m}\right| .
$$

For the far-field configuration, the corresponding dyadic Green function and scattered electric field approximations are investigated. For a fixed $\mathbf{y}$, the following far-field approximation holds:

$$
g(\mathbf{x}, \mathbf{y})=\frac{\mathrm{e}^{\mathrm{i} k_{0} \mathbf{x}}}{4 \pi \mathbf{x}} \mathrm{e}^{-\mathrm{i} \hat{\mathbf{x}} \cdot \mathbf{y}}+O\left(\frac{1}{\mathbf{x}^{2}}\right),
$$

and

$$
\underline{\mathbf{G}}(\mathbf{x}, \mathbf{y})=\frac{\mathrm{e}^{\mathrm{i} k_{0} \mathbf{x}}}{4 \pi \mathbf{x}} \mathrm{e}^{-\mathrm{i} k_{0} \hat{\mathbf{x}} \cdot \mathbf{y}} \Delta(\hat{\mathbf{x}})+O\left(\frac{1}{\mathbf{x}^{2}}\right),
$$

where $\Delta(\hat{\mathbf{x}})=\underline{\mathbf{I}}_{3}-\hat{\mathbf{x}} \otimes \hat{\mathbf{x}}$. Then the far-field pattern of $\underline{\mathbf{G}}(\mathbf{x}, \mathbf{y})$ is given by $\mathrm{r}$

$$
\underline{\mathbf{G}}_{\infty}(\hat{\mathbf{x}}, \mathbf{y}):=\mathrm{e}^{-\mathrm{i} k_{0} \hat{\mathbf{x}} \cdot \mathbf{y}} \Delta(\hat{\mathbf{x}}),
$$

and the incident plane wave field due to a dipole can expressed as

$$
\mathbf{E}_{\infty}^{i}(\mathbf{x}, \hat{\mathbf{y}}):=\mathrm{i} \omega_{0} \mu_{0} I l \underline{\mathbf{G}}_{\infty}(\mathbf{x}, \hat{\mathbf{y}}) \cdot \hat{\mathbf{p}}^{t}
$$

where $-\hat{\mathbf{y}}$ is the impinging direction and $\hat{\mathbf{p}}^{t}$ is its polarization on $\mathbb{S}^{2}$. Hence, the total electric field $\mathbf{E}^{s}(\mathbf{x}, \hat{\mathbf{y}})$ has the far-field pattern $\mathbf{E}_{\infty}(\hat{\mathbf{x}}, \hat{\mathbf{y}})$ such that

$$
\mathbf{E}^{s}(\mathbf{x}, \hat{\mathbf{y}})=\frac{\mathrm{e}^{\mathrm{i} k_{0} \mathbf{x}}}{4 \pi \mathbf{x}} \mathbf{E}_{\infty}(\hat{\mathbf{x}}, \hat{\mathbf{y}})+O\left(\frac{1}{\mathbf{x}^{2}}\right),
$$


uniformly into all directions $\hat{\mathbf{x}}=\mathbf{x} /|\mathbf{x}| \in \mathbb{S}^{2}$ as $|\mathbf{x}| \rightarrow \infty$

Following the same path than the near-field formulation, the far-field pattern $\mathbf{E}_{\infty}(\hat{\mathbf{x}}, \hat{\mathbf{y}})$ has following asymptotic expansion formula.

Lemma 2.2 (Asymptotic formula of far-field pattern). Assume that all inhomogeneities are dielectric and sufficiently small $\left(\alpha_{m} \sqrt{\varepsilon_{m} / \varepsilon_{0}} \ll \lambda_{0} / 2\right)$. Then, $\mathbf{E}_{\infty}(\hat{\mathbf{x}}, \hat{\mathbf{y}})$ has the following asymptotic expansion formula:

$$
\mathbf{E}_{\infty}(\hat{\mathbf{x}}, \hat{\mathbf{y}})=\omega_{0}^{2} \mu_{0} \sum_{m=1}^{M} \alpha_{m}^{3}\left(\varepsilon_{m}-\varepsilon_{0}\right) \underline{\mathbf{G}}_{\infty}\left(\hat{\mathbf{x}}, \mathbf{r}_{m}\right) \underline{\mathbf{M}}\left(\frac{\varepsilon_{m}}{\varepsilon_{0}} ; \mathbf{D}_{m}\right) \mathbf{E}_{\infty}^{i}\left(\mathbf{r}_{m}, \hat{\mathbf{y}}\right)+O\left(\alpha^{4}\right)
$$

where $\alpha:=\max \left\{\alpha_{m}, m=1,2 \cdots, M\right\}$ for a generic incident field $\mathbf{E}_{\infty}^{i}\left(\mathbf{r}_{m}, \hat{\mathbf{y}}\right)$. Introducing the far-field pattern of the dyadic Green function (16) and the expression of plane wave field due to the dipole (17) in (19) leads to

$$
\mathbf{E}_{\infty}(\hat{\mathbf{x}}, \hat{\mathbf{y}})=\mathrm{i} \omega_{0} \mu_{0} k_{0}^{2} I l \sum_{m=1}^{M} \alpha_{m}^{3}\left(\frac{\varepsilon_{m}-\varepsilon_{0}}{\varepsilon_{0}}\right) \mathrm{e}^{-\mathrm{i} k_{0}(\hat{\mathbf{x}}+\hat{\mathbf{y}}) \cdot \mathbf{r}_{m}}\left[\Delta\left(\hat{\mathbf{x}}_{n}\right) \underline{\mathbf{M}}\left(\frac{\varepsilon_{m}}{\varepsilon_{0}} ; \mathbf{D}_{m}\right) \Delta(\hat{\mathbf{y}}) \cdot \hat{\mathbf{p}}^{t}\right]+O\left(\alpha^{4}\right),
$$

for an incident plane wave of impinging direction $-\hat{\mathbf{y}}$ and polarization $\hat{\mathbf{p}}^{t}$.

Following the same path that for the near-field case (20) can be specialized in the case of spherical using (12) leading to

$$
\mathbf{E}_{\infty}(\hat{\mathbf{x}}, \hat{\mathbf{y}})=\mathrm{i} \omega_{0} \mu_{0} k_{0}^{2} I l \sum_{m=1}^{M} A_{m} \mathrm{e}^{-\mathrm{i} k_{0}(\hat{\mathbf{x}}+\hat{\mathbf{y}}) \cdot \mathbf{r}_{m}}\left[\Delta\left(\hat{\mathbf{x}}_{n}\right) \Delta(\hat{\mathbf{y}}) \cdot \hat{\mathbf{p}}^{t}\right]+O\left(\alpha^{5}\right)
$$

\section{Direct sampling method in near-field case}

\subsection{Introduction of direct sampling method and its structure analysis}

The direct sampling method has been introduced in [32] and only the main equations with our notation will be re-called in the follows. On the basis of the relation ((15) in [32])

$$
\left\langle\mathbf{E}^{s}(\mathbf{x}, \mathbf{y}), \underline{\mathbf{G}}(\mathbf{x}, \mathbf{z}) \cdot \hat{\mathbf{q}}\right\rangle_{L^{2}\left(\Gamma_{\mathrm{obs}}\right)} \approx \frac{1}{k_{0}} \sum_{m=1}^{M} W_{m} \operatorname{Im}\left(\underline{\mathbf{G}}\left(\mathbf{z}, \mathbf{r}_{m}\right)\right) \cdot \hat{\mathbf{q}},
$$

the indicator function of DSM ((16) in [32]) in the case of a single impinging source located at $\mathbf{y}$ with $\hat{\mathbf{p}}^{t}$ as polarization direction is defined by

$$
\mathcal{I}_{\mathrm{DSM}}(\mathbf{z} ; \mathbf{y}, \hat{\mathbf{q}}):=\frac{\left|\left\langle\mathbf{E}^{s}(\mathbf{x}, \mathbf{y}), \underline{\mathbf{G}}(\mathbf{x}, \mathbf{z}) \cdot \hat{\mathbf{q}}\right\rangle_{L^{2}\left(\Gamma_{\mathrm{obs}}\right)}\right|}{\left\|\mathbf{E}^{s}(\mathbf{x}, \mathbf{y})\right\|_{L^{2}\left(\Gamma_{\mathrm{obs}}\right)}\|\underline{\mathbf{G}}(\mathbf{x}, \mathbf{z}) \cdot \hat{\mathbf{q}}\|_{L^{2}\left(\Gamma_{\mathrm{obs}}\right)}},
$$

and for $L$ dipoles impinging directions, it is given by

$$
\mathcal{I}_{\mathrm{DSM}}(\mathbf{z}):=\frac{1}{L} \sum_{l=1}^{L} \mathcal{I}_{\mathrm{DSM}}\left(\mathbf{z} ; \mathbf{y}_{l}, \hat{\mathbf{q}}_{l}\right)
$$

for taking into account each incident field. Here, $\underline{\mathbf{G}}(\mathbf{x}, \mathbf{z}) \cdot \hat{\mathbf{q}}$ is a test dipole with polarization along $\hat{\mathbf{q}}$ and related to receivers. Equation (22) shows that $\mathcal{I}_{\mathrm{DSM}}(\mathbf{z} ; \mathbf{y}, \hat{\mathbf{q}}) \approx 1$ if $\mathbf{z}=\mathbf{r}_{m} \in \tau_{m}$, otherwise $\mathcal{I}_{\mathrm{DSM}}(\mathbf{z} ; \mathbf{y}, \hat{\mathbf{q}}) \nsim 1$ thanks to the oscillation property of dyadic Green function. However, we observed that the choice of the test polarization vector $\hat{\mathbf{q}}$ is a key parameter for the proper reconstruction of the inhomogeneities. So further analysis is necessary to verify the full structure of DSM indicator function to identify the relation of the result of DSM and the choice of polarization. Note that the authors of [32] proposed the guidelines for choosing $\hat{\mathbf{q}}$ such that $\hat{\mathbf{q}}=\hat{\mathbf{p}}^{t}$, but no theoretical reason was not yet provided. So the validity of their suggestion will be proved in this work based on our analysis.

Thanks to the use of the asymptotic formula of scattered field introduced in Lemma 2.2, an representation formula of the DSM indicator function $\mathcal{I}_{\mathrm{DSM}}(\mathbf{z} ; \mathbf{y}, \hat{\mathbf{q}})$ is elucidated in the case of an incident field due to a dipole placed in $\mathbf{y}$ and polarized along $\hat{\mathbf{p}}^{t}$. The derivation is proposed as the following. 
Theorem 3.1. Assume that the total number of receiver directions $N$ is sufficiently large and each inhomogeneity is enough small $\left(\alpha_{m} \sqrt{\varepsilon_{m} / \varepsilon_{9}} \ll 0.5 \lambda_{0}\right)$. Then, the DSM indicator function has following representation formula.

$$
\mathcal{I}_{\mathrm{DSM}}(\mathbf{z} ; \mathbf{y}, \hat{\mathbf{q}})=\frac{\left|\mathcal{L}_{1}(\mathbf{z} ; \mathbf{y}, \hat{\mathbf{q}})\right|}{\max _{\mathbf{z} \in \Omega}\left|\mathcal{L}_{1}(\mathbf{z} ; \mathbf{y}, \hat{\mathbf{q}})\right|}
$$

where

$$
\mathcal{L}_{1}(\mathbf{z} ; \mathbf{y}, \hat{\mathbf{q}})=\sum_{m=1}^{M} A_{m}\left[C_{1}\left(\mathrm{j}_{0}\left(k_{0}\left|\mathbf{r}_{m}-\mathbf{z}\right|\right)-\frac{\mathrm{j}_{1}\left(k_{0}\left|\mathbf{r}_{m}-\mathbf{z}\right|\right)}{k_{0}\left|\mathbf{r}_{m}-\mathbf{z}\right|}\right)+C_{2} \frac{\mathrm{j}_{2}\left(k_{0}\left|\mathbf{r}_{m}-\mathbf{z}\right|\right)}{\left|\mathbf{r}_{m}-\mathbf{z}\right|^{2}}\right] .
$$

where $\mathrm{j}_{s}$ is a spherical Bessel function of integer order $s$ and $A_{m}$ is defined in (13) and $C_{1}$ and $C_{2}$ are given by

$$
C_{1}=\left[\hat{\mathbf{p}}^{t} \cdot \hat{\mathbf{q}}\right] Q_{1}\left(\mathbf{y}, \mathbf{r}_{m}\right)-\frac{\left[\hat{\mathbf{p}}^{t} \cdot\left(\mathbf{r}_{m}-\mathbf{y}\right)\right]\left[\hat{\mathbf{q}} \cdot\left(\mathbf{r}_{m}-\mathbf{y}\right)\right]}{\left|\mathbf{r}_{m}-\mathbf{y}\right|^{2}} Q_{2}\left(\mathbf{y}, \mathbf{r}_{m}\right)
$$

and

$$
\begin{aligned}
C_{2}=\frac{\left[\hat{\mathbf{q}} \cdot\left(\mathbf{r}_{m}-\mathbf{z}\right)\right]\left[\hat{\mathbf{p}}^{t} \cdot\left(\mathbf{r}_{m}-\mathbf{y}\right)\right]\left[\left(\mathbf{r}_{m}-\mathbf{y}\right) \cdot\left(\mathbf{r}_{m}-\mathbf{z}\right)\right]}{\left|\mathbf{r}_{m}-\mathbf{y}\right|^{2}} & Q_{2}\left(\mathbf{y}, \mathbf{r}_{m}\right) \\
& -\frac{\left[\hat{\mathbf{p}}^{t} \cdot\left(\mathbf{r}_{m}-\mathbf{z}\right)\right]\left[\hat{\mathbf{q}} \cdot\left(\mathbf{r}_{m}-\mathbf{z}\right)\right]}{\left|\mathbf{r}_{m}-\mathbf{y}\right|^{2}} Q_{1}\left(\mathbf{y}, \mathbf{r}_{m}\right),
\end{aligned}
$$

respectively. Here, $Q_{1}\left(\mathbf{y}, \mathbf{r}_{m}\right)$ and $Q_{2}\left(\mathbf{y}, \mathbf{r}_{m}\right)$ are defined by

$$
Q_{1}\left(\mathbf{y}, \mathbf{r}_{m}\right)=g\left(\mathbf{y}, \mathbf{r}_{m}\right)-\frac{g\left(\mathbf{y}, \mathbf{r}_{m}\right)}{k_{0}^{2}\left|\mathbf{r}_{m}-\mathbf{y}\right|^{2}}+\frac{\mathrm{i} g\left(\mathbf{y}, \mathbf{r}_{m}\right)}{k_{0}\left|\mathbf{r}_{m}-\mathbf{y}\right|},
$$

and

$$
Q_{2}\left(\mathbf{y}, \mathbf{r}_{m}\right)=g\left(\mathbf{y}, \mathbf{r}_{m}\right)-\frac{3 g\left(\mathbf{y}, \mathbf{r}_{m}\right)}{k_{0}^{2}\left|\mathbf{r}_{m}-\mathbf{y}\right|^{2}}+\frac{3 \mathrm{i} g\left(\mathbf{y}, \mathbf{r}_{m}\right)}{k_{0}\left|\mathbf{r}_{m}-\mathbf{y}\right|} .
$$

Proof. Using the asymptotic formula of scattered field (13) within the denominator of (23) leads to

$$
\begin{aligned}
\left\langle\mathbf{E}^{s}(\mathbf{x}, \mathbf{y}), \underline{\mathbf{G}}(\mathbf{x}, \mathbf{z}) \cdot \hat{\mathbf{q}}\right\rangle_{L^{2}\left(\Gamma_{\mathrm{obs}}\right)} & =\mathrm{i} \omega_{0} \mu_{0} k_{0}^{2} I l \sum_{m=1}^{M} A_{m}\left\langle\underline{\mathbf{G}}\left(\mathbf{x}, \mathbf{r}_{m}\right) \underline{\mathbf{G}}\left(\mathbf{r}_{m}, \mathbf{y}\right) \cdot \hat{\mathbf{p}}^{t}, \underline{\mathbf{G}}(\mathbf{x}, \mathbf{z}) \cdot \hat{\mathbf{q}}\right\rangle_{L^{2}\left(\Gamma_{\mathrm{obs}}\right)} \\
& \approx \mathrm{i} \omega_{0} \mu_{0} k_{0} I l \sum_{m=1}^{M} A_{m}\left(\underline{\mathbf{G}}\left(\mathbf{r}_{m}, \mathbf{y}\right) \cdot \hat{\mathbf{p}}^{t}\right) \cdot\left(\operatorname{Im}\left(\underline{\mathbf{G}}\left(\mathbf{z}, \mathbf{r}_{m}\right)\right) \cdot \hat{\mathbf{q}}\right) .
\end{aligned}
$$

To go from (31) to (32) the following relationship (see [32] for the demonstration)

$$
\left\langle\underline{\mathbf{G}}\left(\mathbf{x}, \mathbf{z}_{1}\right) \cdot \mathbf{p}, \underline{\mathbf{G}}\left(\mathbf{x}, \mathbf{z}_{2}\right) \cdot \mathbf{q}\right\rangle_{L^{2}\left(\Gamma_{\mathrm{obs}}\right)} \approx \frac{1}{k_{0}} \mathbf{p} \cdot \operatorname{Im}\left(\underline{\mathbf{G}}\left(\mathbf{z}_{1}, \mathbf{z}_{2}\right) \cdot \mathbf{q}\right)
$$

is used, where $\mathbf{p} \in \mathbb{C}^{3}, \mathbf{q} \in \mathbb{S}^{2}$ where $\mathbb{S}^{2}$ is unit sphere in $\mathbb{R}^{3}$.

The explicit form of $\underline{\mathbf{G}}\left(\mathbf{r}_{m}, \mathbf{y}\right) \hat{\mathbf{p}}^{t}$ can be represented by

$$
\begin{aligned}
& \left(\underline{\mathbf{G}}\left(\mathbf{r}_{m}, \mathbf{y}\right) \cdot \hat{\mathbf{p}}^{t}\right)_{s}=p_{s}^{t}\left[g\left(\mathbf{y}, \mathbf{r}_{m}\right)-\frac{g\left(\mathbf{y}, \mathbf{r}_{m}\right)}{k_{0}^{2}\left|\mathbf{r}_{m}-\mathbf{y}\right|^{2}}+\frac{\mathrm{i} g\left(\mathbf{y}, \mathbf{r}_{m}\right)}{k_{0}\left|\mathbf{r}_{m}-\mathbf{y}\right|}\right] \\
& -\left(\mathbf{r}_{m}-\mathbf{y}\right)_{s} \frac{\hat{\mathbf{p}}^{t} \cdot\left(\mathbf{r}_{m}-\mathbf{y}\right)}{\left|\mathbf{r}_{m}-\mathbf{y}\right|^{2}}\left[g\left(\mathbf{y}, \mathbf{r}_{m}\right)-\frac{3 g\left(\mathbf{y}, \mathbf{r}_{m}\right)}{k_{0}^{2}\left|\mathbf{r}_{m}-\mathbf{y}\right|^{2}}+\frac{3 \mathrm{i} g\left(\mathbf{y}, \mathbf{r}_{m}\right)}{k_{0}\left|\mathbf{r}_{m}-\mathbf{y}\right|}\right], \\
& =p_{s}^{t} Q_{1}\left(\mathbf{y}, \mathbf{r}_{m}\right)-\left(\mathbf{r}_{m}-\mathbf{y}\right)_{s} \frac{\hat{\mathbf{p}}^{t} \cdot\left(\mathbf{r}_{m}-\mathbf{y}\right)}{\left|\mathbf{r}_{m}-\mathbf{y}\right|^{2}} Q_{2}\left(\mathbf{y}, \mathbf{r}_{m}\right), s=1,2,3,
\end{aligned}
$$

where $Q_{1}\left(\mathbf{y}, \mathbf{r}_{m}\right)$ and $Q_{2}\left(\mathbf{y}, \mathbf{r}_{m}\right)$ are defined (29) and (30), respectively. Similarly, the form of $\operatorname{Im}\left(\underline{\mathbf{G}}\left(\mathbf{z}, \mathbf{r}_{m}\right)\right)$. 
$\hat{\mathbf{q}}$ is as follows

$$
\begin{aligned}
\left(\operatorname{Im}\left(\underline{\mathbf{G}}\left(\mathbf{z}, \mathbf{r}_{m}\right)\right) \cdot \hat{\mathbf{q}}\right)_{s}= & \frac{\hat{p}_{s}^{r}}{4 \pi\left|\mathbf{r}_{m}-\mathbf{z}\right|}\left[\sin \left(k_{0}\left|\mathbf{r}_{m}-\mathbf{z}\right|\right)-\frac{\sin \left(k_{0}\left|\mathbf{r}_{m}-\mathbf{z}\right|\right)}{k_{0}^{2}\left|\mathbf{r}_{m}-\mathbf{z}\right|^{2}}+\frac{\cos \left(k_{0}\left|\mathbf{r}_{m}-\mathbf{z}\right|\right)}{k_{0}\left|\mathbf{r}_{m}-\mathbf{z}\right|}\right] \\
& -\left(\mathbf{r}_{m}-\mathbf{z}\right)_{s} \frac{\hat{\mathbf{q}} \cdot\left(\mathbf{r}_{m}-\mathbf{z}\right)}{4 \pi\left|\mathbf{r}_{m}-\mathbf{z}\right|^{3}} \\
\quad \times & {\left[\sin \left(k_{0}\left|\mathbf{r}_{m}-\mathbf{z}\right|\right)-\frac{3 \sin \left(k_{0}\left|\mathbf{r}_{m}-\mathbf{z}\right|\right)}{k_{0}^{2}\left|\mathbf{r}_{m}-\mathbf{z}\right|^{2}}+\frac{3 \cos \left(k_{0}\left|\mathbf{r}_{m}-\mathbf{z}\right|\right)}{k_{0}\left|\mathbf{r}_{m}-\mathbf{z}\right|}\right] } \\
= & \frac{\hat{p}_{s}^{r}}{4 \pi\left|\mathbf{r}_{m}-\mathbf{z}\right|} f_{1}\left(\mathbf{r}_{m}, \mathbf{z}\right)-\left(\mathbf{r}_{m}-\mathbf{z}\right)_{s} \frac{\hat{\mathbf{q}} \cdot\left(\mathbf{r}_{m}-\mathbf{z}\right)}{4 \pi\left|\mathbf{r}_{m}-\mathbf{z}\right|^{3}} f_{2}\left(\mathbf{r}_{m}, \mathbf{z}\right)
\end{aligned}
$$

where

$$
\begin{aligned}
f_{1}\left(\mathbf{r}_{m}, \mathbf{z}\right) & =\left[\sin \left(k_{0}\left|\mathbf{r}_{m}-\mathbf{z}\right|\right)-\frac{\sin \left(k_{0}\left|\mathbf{r}_{m}-\mathbf{z}\right|\right)}{k_{0}^{2}\left|\mathbf{r}_{m}-\mathbf{z}\right|^{2}}+\frac{\cos \left(k_{0}\left|\mathbf{r}_{m}-\mathbf{z}\right|\right)}{k_{0}\left|\mathbf{r}_{m}-\mathbf{z}\right|}\right] \frac{1}{4 \pi\left|\mathbf{r}_{m}-\mathbf{z}\right|}, \\
& =\frac{k_{0}}{4 \pi}\left[\mathrm{j}_{0}\left(k_{0}\left|\mathbf{r}_{m}-\mathbf{z}\right|\right)-\frac{\mathrm{j}_{1}\left(k_{0}\left|\mathbf{r}_{m}-\mathbf{z}\right|\right)}{k_{0}\left|\mathbf{r}_{m}-\mathbf{z}\right|}\right], \\
f_{2}\left(\mathbf{r}_{m}, \mathbf{z}\right) & =\left[\sin \left(k_{0}\left|\mathbf{r}_{m}-\mathbf{z}\right|\right)-\frac{3 \sin \left(k_{0}\left|\mathbf{r}_{m}-\mathbf{z}\right|\right)}{k_{0}^{2}\left|\mathbf{r}_{m}-\mathbf{z}\right|^{2}}+\frac{3 \cos \left(k_{0}\left|\mathbf{r}_{m}-\mathbf{z}\right|\right)}{k_{0}\left|\mathbf{r}_{m}-\mathbf{z}\right|}\right] \frac{1}{4 \pi\left|\mathbf{r}_{m}-\mathbf{z}\right|^{3}} \\
& =-\frac{k_{0}}{4 \pi} \frac{\mathrm{j}_{2}\left(k_{0}\left|\mathbf{r}_{m}-\mathbf{z}\right|\right)}{\left|\mathbf{r}_{m}-\mathbf{z}\right|^{2}} .
\end{aligned}
$$

Here, the definitions of first kind of spherical Bessel functions are used to go from (38) to (39) and from (40) to (41), respectively.

The combination of (35) and (37) for $s=1,2,3$, leads to

$$
\begin{aligned}
&\left(\underline{\mathbf{G}}\left(\mathbf{r}_{m}, \mathbf{y}\right) \cdot \hat{\mathbf{p}}^{t}\right) \cdot\left(\operatorname{Im}\left(\underline{\mathbf{G}}\left(\mathbf{z}, \mathbf{r}_{m}\right)\right) \cdot \hat{\mathbf{q}}\right) \\
&= \sum_{s=1}^{3}\left(\underline{\mathbf{G}}\left(\mathbf{r}_{m}, \mathbf{y}\right) \hat{\mathbf{p}}^{t}\right)_{s}\left(\operatorname{Im}\left(\underline{\mathbf{G}}\left(\mathbf{z}, \mathbf{r}_{m}\right)\right) \hat{\mathbf{q}}\right)_{s} \\
&=\left\{\left[\hat{\mathbf{p}}^{t} \cdot \hat{\mathbf{q}}\right] Q_{1}\left(\mathbf{y}, \mathbf{r}_{m}\right)-\frac{\left[\hat{\mathbf{p}}^{t} \cdot\left(\mathbf{r}_{m}-\mathbf{y}\right)\right]\left[\hat{\mathbf{q}} \cdot\left(\mathbf{r}_{m}-\mathbf{y}\right)\right]}{\left|\mathbf{r}_{m}-\mathbf{y}\right|^{2}} Q_{2}\left(\mathbf{y}, \mathbf{r}_{m}\right)\right\} f_{1}\left(\mathbf{r}_{m}, \mathbf{z}\right) \\
&+\left\{\frac{\left[\hat{\mathbf{q}} \cdot\left(\mathbf{r}_{m}-\mathbf{z}\right)\right]\left[\hat{\mathbf{p}}^{t} \cdot\left(\mathbf{r}_{m}-\mathbf{y}\right)\right]\left[\left(\mathbf{r}_{m}-\mathbf{y}\right) \cdot\left(\mathbf{r}_{m}-\mathbf{z}\right)\right]}{\left|\mathbf{r}_{m}-\mathbf{y}\right|^{2}} Q_{2}\left(\mathbf{y}, \mathbf{r}_{m}\right)\right. \\
&\left.-\frac{\left[\hat{\mathbf{p}}^{t} \cdot\left(\mathbf{r}_{m}-\mathbf{z}\right)\right]\left[\hat{\mathbf{q}} \cdot\left(\mathbf{r}_{m}-\mathbf{z}\right)\right]}{\left|\mathbf{r}_{m}-\mathbf{y}\right|^{2}} Q_{1}\left(\mathbf{y}, \mathbf{r}_{m}\right)\right\} f_{2}\left(\mathbf{r}_{m}, \mathbf{z}\right)
\end{aligned}
$$

After some manipulations and with the expansion of $f_{1}\left(\mathbf{r}_{m}, \mathbf{z}\right)$ and $f_{2}\left(\mathbf{r}_{m}, \mathbf{z}\right)$ as combination of the spherical Bessel functions as defined in (39) and (41), the final expression is obtained as

$$
\left|\left\langle\mathbf{E}^{s}(\mathbf{x}, \mathbf{y}), \underline{\mathbf{G}}(\mathbf{x}, \mathbf{z}) \hat{\mathbf{q}}\right\rangle_{L^{2}\left(\Gamma_{\mathrm{obs}}\right)}\right| \approx\left|\sum_{m=1}^{M} A_{m}\left[C_{1}\left(\mathrm{j}_{0}\left(k_{0}\left|\mathbf{r}_{m}-\mathbf{z}\right|\right)-\frac{\mathrm{j}_{1}\left(k_{0}\left|\mathbf{r}_{m}-\mathbf{z}\right|\right)}{k_{0}\left|\mathbf{r}_{m}-\mathbf{z}\right|}\right)+C_{2} \frac{\mathrm{j}_{2}\left(k_{0}\left|\mathbf{r}_{m}-\mathbf{z}\right|\right)}{\left|\mathbf{r}_{m}-\mathbf{z}\right|^{2}}\right]\right|,
$$

where $C_{1}$ and $C_{2}$ are given by (27) and (28) and where the constant $\frac{\mathrm{i} \omega_{0} \mu_{0} I l k_{0}^{2}}{4 \pi}$ is omitted since canceled in the final expression thanks to the Hölder inequality

$$
\left|\langle\mathbf{f}, \mathbf{g}\rangle_{L^{2}\left(\Gamma_{\mathrm{obs}}\right)}\right| \leq\|\mathbf{f}\|_{L^{2}\left(\Gamma_{\mathrm{obs}}\right)}\|\mathbf{g}\|_{L^{2}\left(\Gamma_{\mathrm{obs}}\right)}
$$

which completes the proof.

Remark 3.1 (Properties of DSM with near-field data). We investigate the properties of $\mathcal{I}_{\mathrm{DSM}}(\mathbf{z} ; \mathbf{y}, \hat{\mathbf{q}})$ from the result in Theorem 3.1 as follows.

1. Due to the properties of spherical Bessel function of integer orders in Figure 2, we have $\mathrm{j}_{0}\left(k_{0} \mid \mathbf{r}_{m}-\right.$ $\mathbf{z} \mid)=1, \mathrm{j}_{1}\left(k_{1}\left|\mathbf{r}_{m}-\mathbf{z}\right|\right)=0$ and $\mathrm{j}_{2}\left(k_{0}\left|\mathbf{r}_{m}-\mathbf{z}\right|\right)=0$ if $\mathbf{z}=\mathbf{r}_{m}, m=1,2, \cdots, M$. In other words, each inhomogeneity can be identified via $\mathcal{I}_{\operatorname{DSM}}(\mathbf{z} ; \mathbf{y}, \hat{\mathbf{q}})$ due to the term of $\mathrm{j}_{0}\left(k_{0}\left|\mathbf{r}_{m}-\mathbf{z}\right|\right)$. But unexpected artifacts will be discovered in the map of $\mathcal{I}_{\mathrm{DSM}}(\mathbf{z} ; \mathbf{y}, \hat{\mathbf{q}})$ because of the remaining terms $\mathrm{j}_{1}\left(k_{0}\left|\mathbf{r}_{m}-\mathbf{z}\right|\right)$ and $\mathrm{j}_{2}\left(k_{0}\left|\mathbf{r}_{m}-\mathbf{z}\right|\right)$. 
2. The physical quantity of the inhomogeneities, e.g., the permittivity and size (which have been concatenated within the complex amplitude $A_{m}$ for defect numbered $m$ ), have an important rule in the map of $\mathcal{I}_{\mathrm{DSM}}(\mathbf{z} ; \mathbf{y}, \hat{\mathbf{q}})$. More specifically, the latter has its maximum values at the location of the inhomogeneity, having the highest complex amplitude $A_{m}$ so that the one with the smallest amplitude might be difficult to be identified. It is an inherent limitation of the DSM approach, as it has been shown the details for the 2D scalar case in our previous work [30].

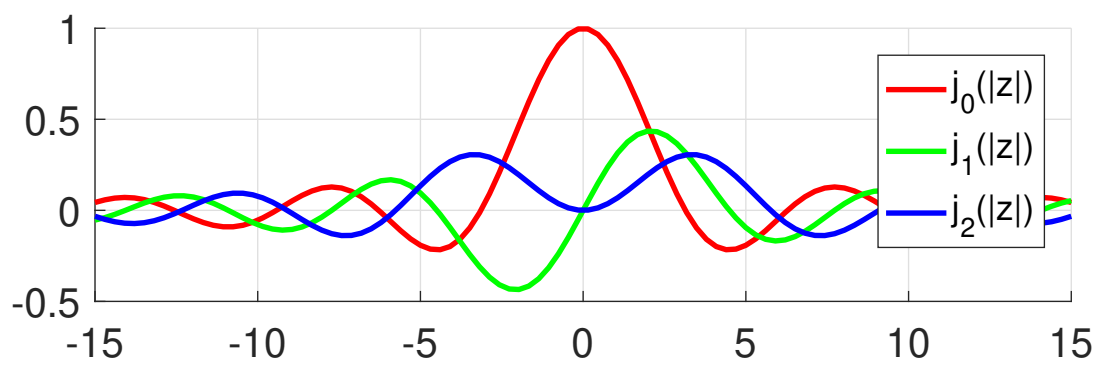

Figure 2: One-dimensional plot of the spherical Bessel function with orders 0, 1, 2

Based on our theoretical result in Theorem 3.1, we can know that choosing $\hat{\mathbf{q}}$ has an important rule for proper reconstruction of support of the inhomogeneities. So a further discussion about choosing $\hat{\mathbf{q}}$ is essential to apply the DSM.

Remark 3.2 (Investigation of choosing proper $\hat{\mathbf{q}}$ ).

1. The results provided by $\mathcal{I}_{\mathrm{DSM}}(\mathbf{z} ; \mathbf{y}, \hat{\mathbf{q}})$ are highly dependent on the choice of the test dipole polarization $\hat{\mathbf{q}} \in \mathbb{S}^{2}$ because the disturbing term could not have effect for imaging if $C_{1}=0$ where $C_{1}$ given by (27) which means that $\mathcal{I}_{\mathrm{DSM}}(\mathbf{z} ; \mathbf{y}, \hat{\mathbf{q}}) \neq 1$ when $\mathbf{z}=\mathbf{r}_{m} \in \tau_{m}$. Hence, we have to choose $\hat{\mathbf{q}}$ at least satisfying $C_{1} \neq 0$, i.e,

$$
\left[\hat{\mathbf{p}}^{t} \cdot \hat{\mathbf{q}}\right] Q_{1}\left(\mathbf{y}, \mathbf{r}_{m}\right) \neq \frac{\left[\hat{\mathbf{p}}^{t} \cdot\left(\mathbf{r}_{m}-\mathbf{y}\right)\right]\left[\hat{\mathbf{q}} \cdot\left(\mathbf{r}_{m}-\mathbf{y}\right)\right]}{\left|\mathbf{r}_{m}-\mathbf{y}\right|^{2}} Q_{2}\left(\mathbf{y}, \mathbf{r}_{m}\right)
$$

So (45) is a necessary condition to identify the inhomogeneities via $\mathcal{I}_{\mathrm{DSM}}(\mathbf{z} ; \mathbf{y}, \hat{\mathbf{q}})$.

2. From our theoretical result (25), the best choice of $\hat{\mathbf{q}}$ for imaging unknown targets with excellent performance is to select $\hat{\mathbf{q}}$ making the large magnitude of $C_{1}$ and small magnitude of $C_{2}$ at the same time. Unfortunately, it is impossible to select it analytically without information about $\tau_{m}$ since both of $C_{1}$ and $C_{2}$ depend on the unknown information $\mathbf{r}_{m}$.

3. Following the pioneer work [33], leading to an enhanced MUSIC algorithm, the polarization of test dipole $\hat{\mathbf{q}}_{\max }$ was chosen to maximize the back-propagation amplitude in each sampling point $\mathbf{z} \in \Omega$. So we suggest an alternative indicator function (DSMP) without any a priori information such as follows:

$$
\mathcal{I}_{\mathrm{DSMP}}(\mathbf{z} ; \mathbf{y})=\max _{\hat{\mathbf{q}} \in \mathbb{S}^{2}}\left\{\mathcal{I}_{\mathrm{DSM}}(\mathbf{z} ; \mathbf{y}, \hat{\mathbf{q}})\right\}
$$

even though more computational cost is required.

Now, we theoretically verify the validation of the guideline for choosing $\hat{\mathbf{q}}$ proposed from the authors of [32] $\left(\hat{\mathbf{q}}=\mathbf{p}^{t}\right)$ through our structure analysis.

Corollary 3.2. Let $\mathbf{r}_{m} \neq \mathbf{y}, m=1,2, \cdots, M$ and $\hat{\mathbf{q}}=\mathbf{p}^{t} \in \mathbb{S}^{2}$. Then, the necessary condition holds.

Proof. Assume that the condition (45) is no longer satisfied when $\hat{\mathbf{q}}=\mathbf{p}^{t}$, i.e.,

$$
\left[\hat{\mathbf{p}}^{t} \cdot \hat{\mathbf{q}}\right] Q_{1}\left(\mathbf{y}, \mathbf{r}_{m}\right)=\frac{\left[\hat{\mathbf{p}}^{t} \cdot\left(\mathbf{r}_{m}-\mathbf{y}\right)\right]\left[\hat{\mathbf{q}} \cdot\left(\mathbf{r}_{m}-\mathbf{y}\right)\right]}{\left|\mathbf{r}_{m}-\mathbf{y}\right|^{2}} Q_{2}\left(\mathbf{y}, \mathbf{r}_{m}\right), \text { where } \hat{\mathbf{q}}=\mathbf{p}^{t}
$$

Then, since $\left|\mathbf{p}^{t}\right|=1$ and $\mathbf{p}^{t} \cdot \mathbf{p}^{t}=1$,

$$
Q_{1}\left(\mathbf{y}, \mathbf{r}_{m}\right)=\left(\cos ^{2} \vartheta\right) Q_{2}\left(\mathbf{y}, \mathbf{r}_{m}\right)
$$


where $\vartheta$ is the between angle of two vectors $\mathbf{p}^{t}$ and $\mathbf{r}_{m}-\mathbf{y}$. The definitions of $Q_{1}\left(\mathbf{y}, \mathbf{r}_{m}\right)$ and $Q_{2}\left(\mathbf{y}, \mathbf{r}_{m}\right)$ defined by (29) and (30), respectively, leads to

$$
g\left(\mathbf{y}, \mathbf{r}_{m}\right)\left[1-\frac{1}{k_{0}^{2}\left|\mathbf{r}_{m}-\mathbf{y}\right|^{2}}+\frac{\mathrm{i}}{k_{0}\left|\mathbf{r}_{m}-\mathbf{y}\right|}\right]=g\left(\mathbf{y}, \mathbf{r}_{m}\right)\left[1-\frac{3}{k_{0}^{2}\left|\mathbf{r}_{m}-\mathbf{y}\right|^{2}}+\frac{3 \mathrm{i}}{k_{0}\left|\mathbf{r}_{m}-\mathbf{y}\right|}\right] \cos ^{2} \vartheta
$$

which is equivalent to the case that the real and imaginary parts are simultaneously null. Namely,

$$
1-\frac{1}{k_{0}^{2}\left|\mathbf{r}_{m}-\mathbf{y}\right|^{2}}=\left[1-\frac{3}{k_{0}^{2}\left|\mathbf{r}_{m}-\mathbf{y}\right|^{2}}\right] \cos ^{2} \vartheta
$$

and

$$
\frac{1}{k_{0}\left|\mathbf{r}_{m}-\mathbf{y}\right|}=\left[\frac{3}{k_{0}\left|\mathbf{r}_{m}-\mathbf{y}\right|}\right] \cos ^{2} \vartheta
$$

Since $|\mathbf{r} m-\mathbf{y}| \neq 0$, the imaginary part (51) implies to $\cos ^{2} \vartheta=\frac{1}{3}$. By introducing the latter to (50),

$$
1=\frac{1}{3}
$$

which is a contradiction. Therefore, $C_{1} \neq 0$ if $\hat{\mathbf{q}}=\mathbf{p}^{t}$ which is the completeness of the proof.

Above observation shows that the necessary condition (45) is satisfied if $\hat{\mathbf{q}}=\mathbf{p}^{t}$, so the inhomogeneities can be identified via DSM with $\hat{\mathbf{q}}=\mathbf{p}^{t}$ when imaging the small volume spherical targets.

\subsection{Numerical simulations}

In this section, our theoretical results and efficiency of our methods are examined through various numerical simulations. We consider a fixed frequency $f_{0}=749.481 \mathrm{MHz}=c_{0} / \lambda_{0}$ where $\lambda_{0}=0.4 \mathrm{~m}$ is the wavelength and $c_{0}$ the speed of light. FEKO is used to compute the fields scattered by various obstacles illuminated by a single fixed dipole placed at $\mathbf{y}=\left(2.5 \lambda_{0}, 90^{\circ}, 0^{\circ}\right)$ and polarized along $z$-axis, where $(r, \theta, \phi)$ indicate the radial distance, polar angle, and azimuthal angle in spherical coordinate system and polarized along $\hat{\theta}$. The three components $x, y, z$ of the scattered fields are measured at the receiver location defined as $\mathbf{x}=\left(r^{r}, \theta^{r}, \phi^{r}\right)$, i.e., $\mathbf{E}^{s}(\mathbf{x}, \mathbf{y})=\left[E_{x}^{s}(\mathbf{x}, \mathbf{y}), E_{y}^{s}(\mathbf{x}, \mathbf{y}), E_{z}^{s}\left(\mathbf{x}, \mathbf{y} ; k_{0}\right)\right]$. Here, $r^{r}=2.5 \lambda_{0}$, $\theta_{n_{\theta}}^{r}=10 n_{\theta}{ }^{\circ}, n_{\theta}=1,2, \cdots, 17, \phi_{n_{\phi}}^{r}=10\left(n_{\phi}-1\right)^{\circ}, n_{\phi}=1,2, \cdots, 36$, i.e., the total number of receivers is $N=N_{\theta} \times N_{\phi}=612$. A 20-dB white Gaussian random noise is added using the MATLAB function awgn. The region of interest (ROI) is defined as a $2.5 \lambda_{0}$-side length cube evenly discretized in $41 \times 41 \times 41$ voxels. To apply our proposal DSMP, we consider $\hat{\mathbf{q}}_{t} \in \mathbb{S}^{2}, t=1,2, \cdots, 201$ that are evenly distributed by the method in [37].

The reconstructions are visualized using volume slice planes and/or isosurface the latter being defined as

$$
\mathcal{V}_{v}=\{\mathbf{z} \in \Omega \mid \mathcal{I}(\mathbf{z}) \geq v\},
$$

where $v$ is an isosurface parameter and $\mathcal{I}$ is either $\mathcal{I}_{\mathrm{DSM}}(\mathbf{z} ; \mathbf{y}, \hat{\mathbf{q}})$ or $\mathcal{I}_{\mathrm{DSMP}}(\mathbf{z} ; \mathbf{y})$. According to [38], we choose the parameter $v$ such as

$$
v=\min _{\mathbf{z} \in \Omega} \mathcal{I}(\mathbf{z})+\rho\left[\max _{\mathbf{z} \in \Omega} \mathcal{I}(\mathbf{z})-\min _{\mathbf{z} \in \Omega} \mathcal{I}(\mathbf{z})\right], \quad 0<\rho<1,
$$

and $\rho$ is chosen based upon the quality of the reconstructions.

In order to compare the accuracy of the results, we adopt the traditional notion of the Jaccard index [39]. The index measures the similarity between two finite samples sets $A$ and $B$, and it is defined as

$$
J(A, B)(\%):=\frac{|A \cap B|}{|A \cup B|} \times 100 .
$$

In our case the Jaccard index is calculated by comparing $\mathcal{I}_{\text {exact }}^{\kappa}(\mathbf{z})$ with various index maps $\mathcal{I}^{\kappa}(\mathbf{z})$ defined as

$$
\mathcal{I}^{\kappa}(\mathbf{z})=\left\{\begin{array}{cll}
\mathcal{I}(\mathbf{z}), & \forall \mathbf{z} & \text { such that } \mathcal{I} \geq \kappa \\
0 & \forall \mathbf{z} & \text { such that } \mathcal{I}<\kappa
\end{array}\right.
$$

where $\kappa$ varies from 0 to 1 and where $\mathcal{I}(\mathbf{z})$ can be $\mathcal{I}_{\mathrm{DSM}}(\mathbf{z} ; \mathbf{y}, \hat{\mathbf{q}})$ or $\mathcal{I}_{\mathrm{DSMP}}(\mathbf{z} ; \mathbf{y})$ and where $\mathcal{I}_{\text {exact }}(\mathbf{z})$ is defined as

$$
\mathcal{I}_{\text {exact }}(\mathbf{z})=\frac{\left|k(\mathbf{z})-k_{0}\right|}{\max \left|k(\mathbf{z})-k_{0}\right|}
$$

Here, $k(\mathbf{z})$ is the wavenumber at the searching point $\mathbf{z} \in \Omega$. The $k_{0}$ is analogously defined in the background medium. 
Example 3.1 (Single small sphere). A small spherical dielectric inhomogeneity of location $\left(\frac{\lambda_{0}}{4}, \frac{\lambda_{0}}{4}, \frac{\lambda_{0}}{4}\right)$, radius $\frac{\lambda_{0}}{10}$ and electric permittivity $\varepsilon=5 \varepsilon_{0}$ is considered.

Figure 3 shows that the accuracy of DSM is highly dependent on the choice of the test dipole polarization, as stated in Remark 3.2. As for example when changing the test dipole polarization in the range of $\hat{\mathbf{q}}$ in $0^{\circ} \leq \theta \leq 140^{\circ}$ for fixed $\phi=45^{\circ}$, the DSM results evolves from a good localization to bad one. We can identify the inhomogeneity via DSM when the polarization is chosen either $\hat{\mathbf{q}}=\mathbf{p}^{t}$ (by the guideline in [32]) or our proposal DSMP in (46), See Figure 4, and Figure 5, respectively. The results show that their accuracy seems to be similar, the Jaccard index identifies that in Figure 6. But additional information (e.g., the polarization of incident dipole) is not used in our proposal with a slightly higher computational cost.
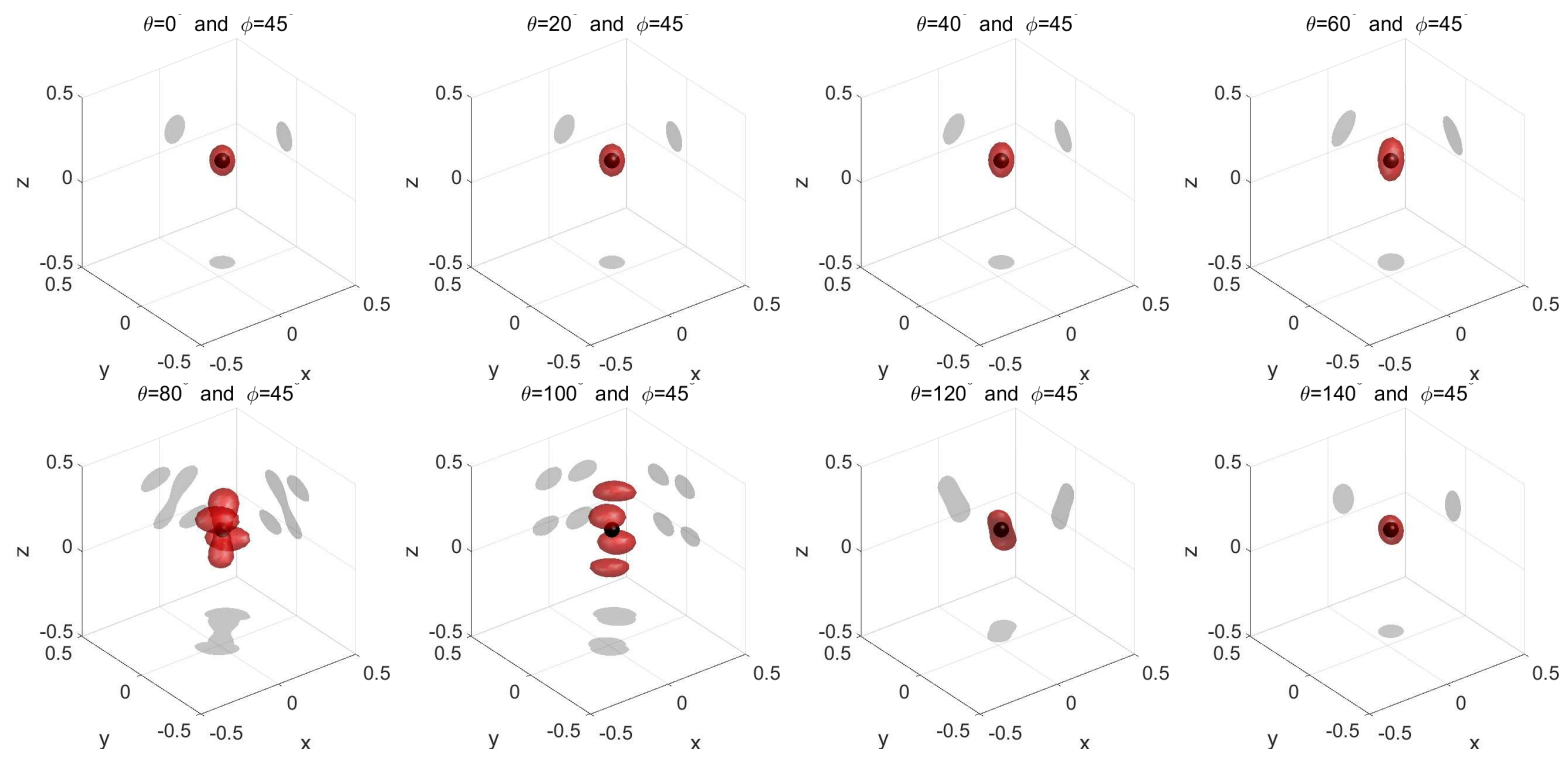

Figure 3: (Example 3.1) Maps of $\mathcal{I}_{\mathrm{DSM}}(\mathbf{z} ; \mathbf{y}, \hat{\mathbf{q}})$ using isosurface with $\rho=0.8$, where $\hat{\mathbf{q}}=(1, \theta, \phi)$

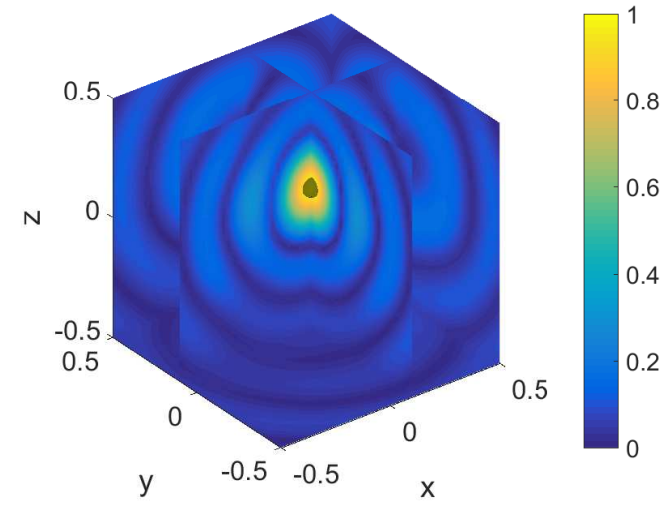

(a) Volume slice with $\mathbf{x}=0.1$ and $\mathbf{y}=0.1$ planes

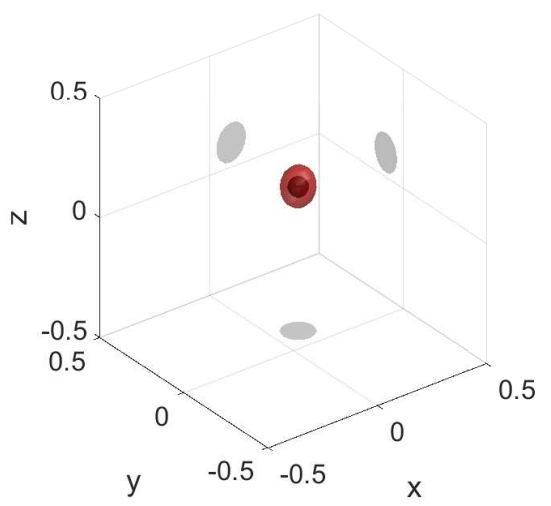

(b) Isosurface with $\rho=0.8$

Figure 4: (Example 3.1) Maps of $\mathcal{I}_{\mathrm{DSM}}(\mathbf{z} ; \mathbf{y}, \hat{\mathbf{q}})$, where $\hat{\mathbf{q}}=\left(1,90^{\circ}, 45^{\circ}\right)=(0,0,1)=\hat{\mathbf{p}}^{t}$

Example 3.2 (Two small spheres with different radii but same permittivity). In the following, the case of two dielectric spheres with different radii $\alpha_{1}=0.12 \lambda_{0}, \alpha_{2}=0.1 \lambda_{0}$ but same electric permittivity $\varepsilon_{m}=5 \varepsilon_{0}, m=1,2$, located at $\mathbf{r}_{1}=\left(\frac{\lambda_{0}}{4}, \frac{\lambda_{0}}{4}, \frac{\lambda_{0}}{4}\right)$ and $\mathbf{r}_{2}=\left(-\frac{\lambda_{0}}{2},-\frac{\lambda_{0}}{4},-\frac{\lambda_{0}}{4},\right)$, respectively is dealt with.

According to Figure 7, the proper choice of the test dipole polarization is a crucial parameter for the efficiency of DSM also in multiple target case. Figures 8, 9, 10 show that both of the selections of polarization provide a good result. Note that the isosurface representation has been drawn using $\rho=0.4$ (instead of $\rho=0.8$ ) to exhibit the localization of $\tau_{2}$. As a matter of fact, $\tau_{2}$ having a smaller size and 


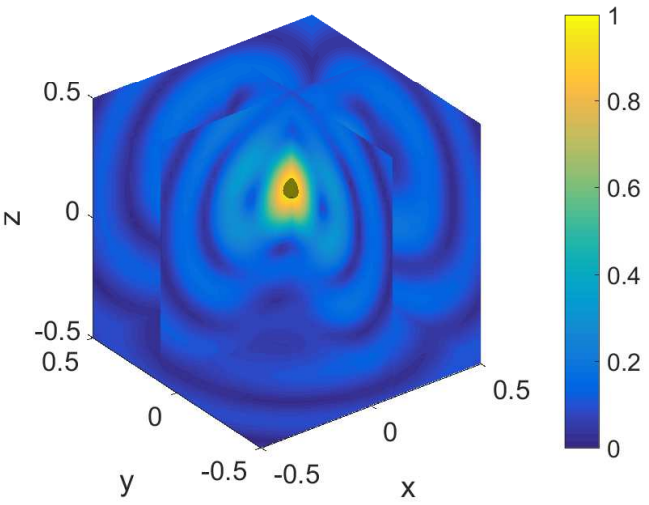

(a) Volume slice with $\mathbf{x}=0.1$ and $\mathbf{y}=0.1$ planes

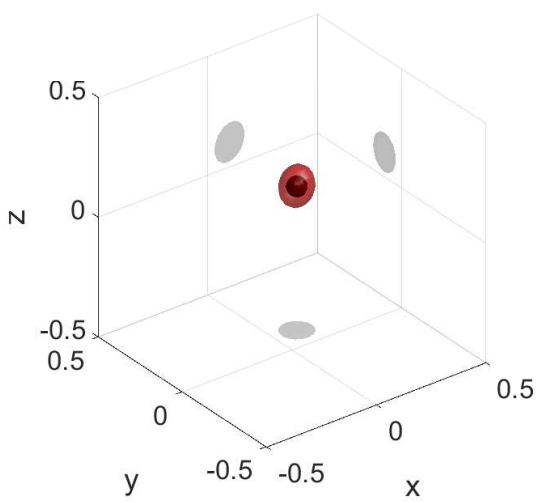

(b) Isosurface with $\rho=0.8$

Figure 5: (Example 3.1) Maps of $\mathcal{I}_{\mathrm{DSMP}}(\mathbf{z} ; \mathbf{y})$

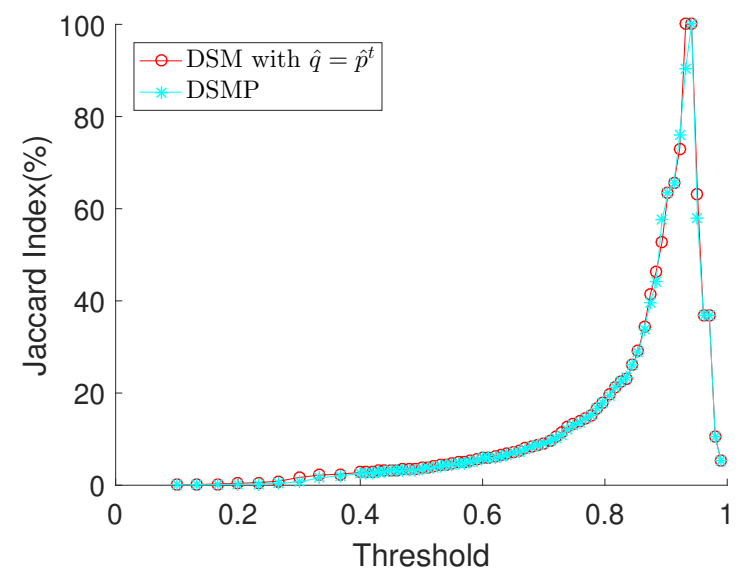

Figure 6: (Example 3.1) Jaccard index of $\mathcal{I}_{\mathrm{DSM}}\left(\mathbf{z} ; \mathbf{y}, \hat{\mathbf{q}}=\hat{\mathbf{p}}^{t}\right)$ and $\mathcal{I}_{\mathrm{DSMP}}(\mathbf{z} ; \mathbf{y})$

being further away from the impinging dipole its signature has a lower value than the one of $\tau_{2}$, as stated in Remark 1 which is an inherent limitation of DSM. We also note that the same phenomenon would have happened in the case of scatterers with the same radii but different permittivity.
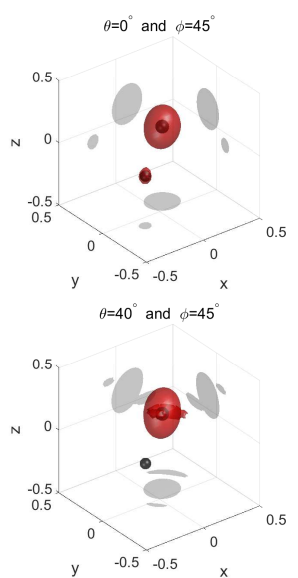
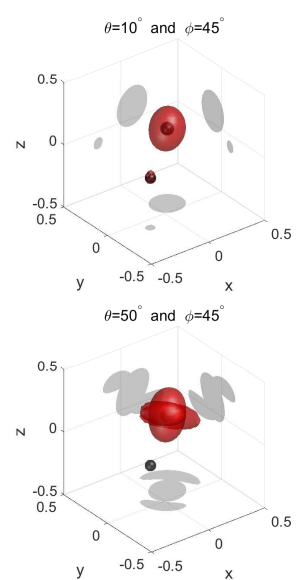

$\theta=20^{\circ}$ and $\phi=45^{\circ}$
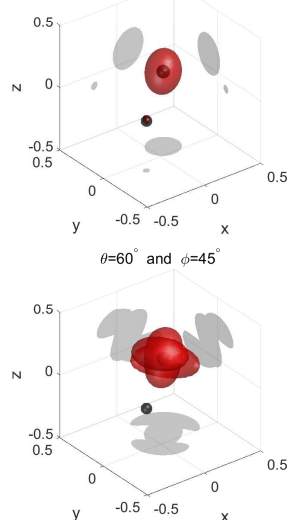

$\theta=30^{\circ}$ and $\phi=45^{\circ}$
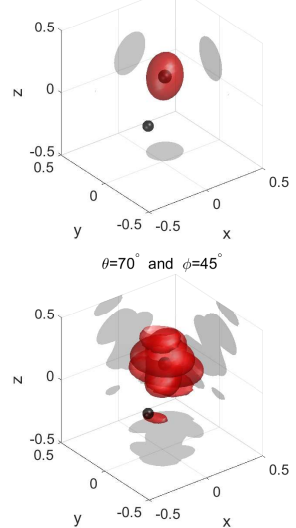

Figure 7: (Example 3.2) Maps of $\mathcal{I}_{\mathrm{DSM}}(\mathbf{z} ; \mathbf{y}, \hat{\mathbf{q}})$ using isosurface with $\rho=0.4$, where $\hat{\mathbf{q}}=(1, \theta, \phi)$ 


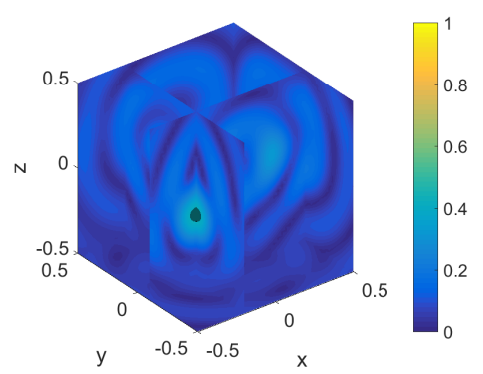

(a) Volume slice with $\mathbf{x}=0.1$ and $\mathbf{y}=$ (b) Volume slice with $\mathbf{x}=-0.2$ and 0.1 planes

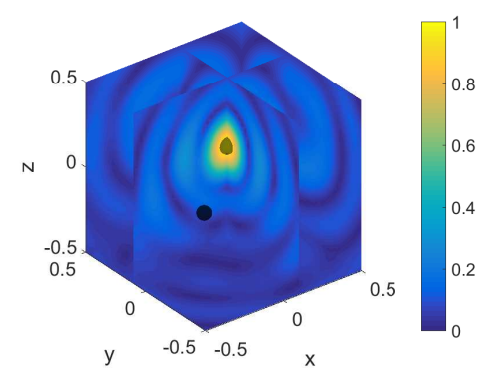

$\mathbf{y}=-0.1$ planes

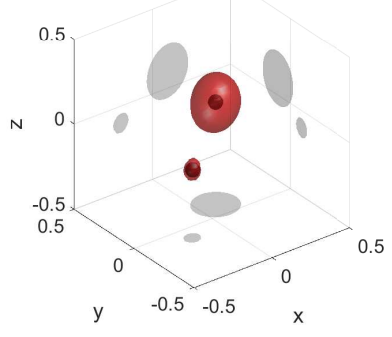

(c) Isosurface with $\rho=0.5$

Figure 8: (Example 3.2) Maps of $\mathcal{I}_{\mathrm{DSM}}(\mathbf{z} ; \mathbf{y}, \hat{\mathbf{q}})$, where $\hat{\mathbf{q}}=\left(1,0^{\circ}, 45^{\circ}\right)=(0,0,1)=\hat{\mathbf{p}}^{t}$

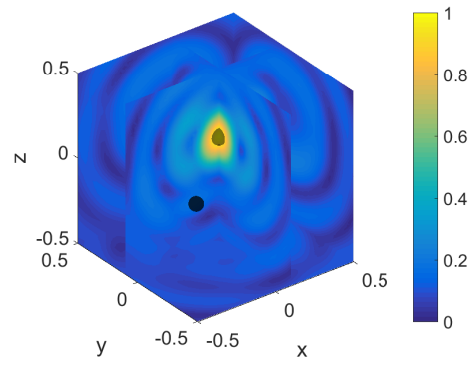

(a) Volume slice with $\mathbf{x}=0.1$ and $\mathbf{y}=$ (b) Volume slice with $\mathbf{x}=-0.2$ and 0.1 planes

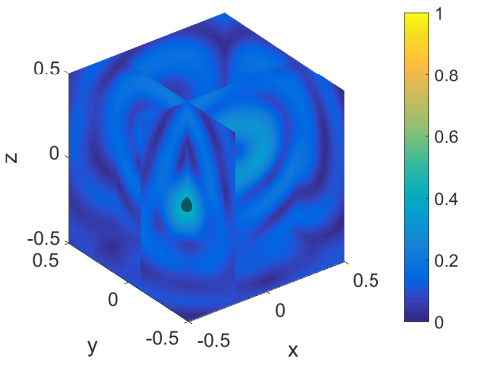

$\mathbf{y}=-0.1$ planes

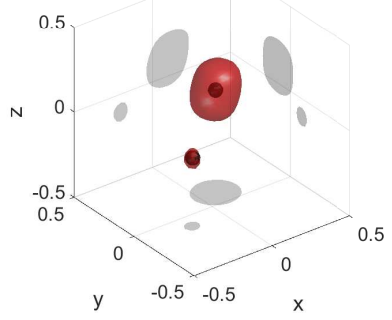

(c) Isosurface with $\rho=0.5$

Figure 9: (Example 3.2) Maps of $\mathcal{I}_{\mathrm{DSMP}}(\mathbf{z} ; \mathbf{y})$

\section{Direct sampling method in far-field case}

\subsection{Introduction of direct sampling method and its structure analysis}

In the following, we will treat the far-field configuration in which the incident field is nothing but a single plane wave $\mathbf{E}_{\infty}^{i}(\hat{\mathbf{x}}, \hat{\mathbf{y}})$ having an impinging direction $-\hat{\mathbf{y}}$ and a polarization $\hat{\mathbf{p}}^{t}$ and the collected data are the far-field pattern $\mathbf{E}_{\infty}(\hat{\mathbf{x}}, \hat{\mathbf{y}})$ as defined (20). The DSM indicator function is then defined as

$$
\mathcal{I}_{\mathrm{DSM}}^{\infty}(\mathbf{z} ; \hat{\mathbf{y}}, \hat{\mathbf{q}}):=\frac{\left|\left\langle\mathbf{E}_{\infty}(\hat{\mathbf{x}}, \hat{\mathbf{y}}), \underline{\mathbf{G}}_{\infty}(\hat{\mathbf{x}}, \mathbf{z}) \cdot \hat{\mathbf{q}}\right\rangle_{L^{2}\left(\mathbb{S}^{2}\right.}\right|}{\left\|\mathbf{E}^{s}(\hat{\mathbf{x}}, \hat{\mathbf{y}})\right\|_{L^{2}\left(\mathbb{S}^{2}\right)}\left\|\underline{\mathbf{G}}_{\infty}(\hat{\mathbf{x}}, \mathbf{z}) \cdot \hat{\mathbf{q}}\right\|_{L^{2}\left(\mathbb{S}^{2}\right)}} .
$$

As usual the DSM indicator function with $L$ multiple impinging directions is given by

$$
\mathcal{I}_{\mathrm{DSM}}^{\infty}(\mathbf{z})=\frac{1}{L} \sum_{l=1}^{L} \mathcal{I}_{\mathrm{DSM}}^{\infty}\left(\mathbf{z} ; \hat{\mathbf{y}}_{l}, \hat{\mathbf{q}}_{l}\right),
$$

where $\mathcal{I}_{\mathrm{DSM}}^{\infty}\left(\mathbf{z} ; \hat{\mathbf{y}}_{l}, \hat{\mathbf{q}}_{l}\right)$ is given in (58) for each $\hat{\mathbf{y}}_{l}, l=1,2, \cdots, L$ (note that (59) is equivalent to (58) if $L=1)$.

Following the same steps as for the near-field case, an analytical formulation of the DSM indicator function is proposed in Theorem 4.4 thanks to the use of the asymptotic formulation of the far-field pattern (20). However, some preliminary results have to be introduced first in order to calculate the following integral equation, a key point to establish an analytical formulation of the DSM indicator function.

$$
\int_{\mathbb{S}^{2}}\left(\underline{\mathbf{G}}_{\infty}\left(\hat{\mathbf{x}}, \mathbf{z}_{1}\right) \cdot \mathbf{p}\right) \cdot\left(\overline{\mathbf{G}_{\infty}\left(\hat{\mathbf{x}}, \mathbf{z}_{2}\right)} \cdot \mathbf{q}\right) \mathrm{d} S(\hat{\mathbf{x}}),
$$

Let us first introduce the well-known Funk-Hecke formula (Lemma 4.1) involving the spherical harmonic function. We will have to deal with and a new lemma (Lemma 4.2) involving either the real part or the imaginary part of the spherical harmonic function (the demonstration of the latter being provided in the appendix) 


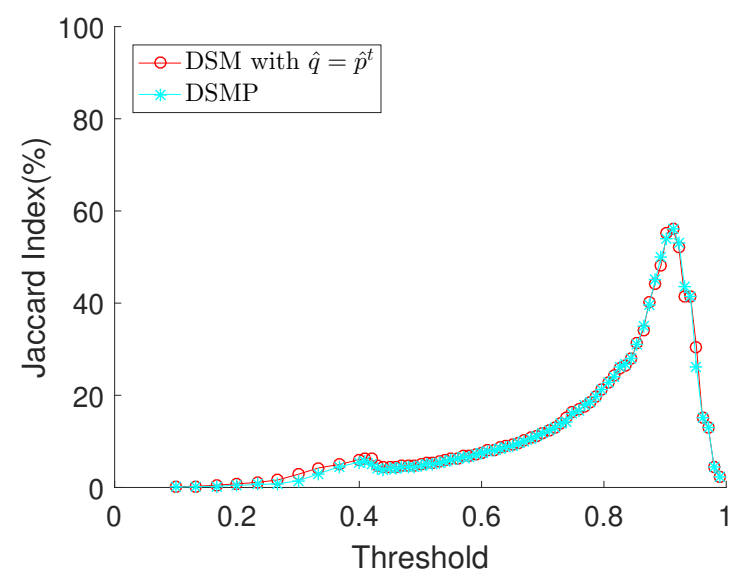

Figure 10: (Example 3.2) Jaccard index of $\mathcal{I}_{\mathrm{DSM}}\left(\mathbf{z} ; \mathbf{y}, \hat{\mathbf{q}}=\hat{\mathbf{p}}^{t}\right)$ and $\mathcal{I}_{\mathrm{DSMP}}(\mathbf{z} ; \mathbf{y})$

Lemma 4.1 (Funk-Hecke formula). For any $f \in L^{2}(-1,1)$ and $\hat{\mathbf{z}}, \hat{\mathbf{x}} \in \mathbb{S}^{2}$, the following formula holds.

$$
\int_{\mathbb{S}^{2}} f(\hat{\mathbf{z}} \cdot \hat{\mathbf{x}}) \mathrm{Y}_{n}^{m}(\hat{\mathbf{x}}) \mathrm{d} S(\hat{\mathbf{x}})=\lambda_{n} \mathrm{Y}_{n}^{m}(\hat{\mathbf{z}}) \text { where } \lambda_{n}=2 \pi \int_{-1}^{1} f(t) \mathrm{P}_{n}(t) \mathrm{d} t
$$

for all $n \in \mathbb{N}$ and $m=-n, \ldots, n$. Here, $\mathrm{Y}_{n}^{m}$ is a spherical harmonic and $\mathrm{P}_{n}$ is a Legendre polynomial.

The details can be found in $[40,41]$ and reference therein. Following the same path than the FunkHecke formula proof founded in [41, Theorem 2.16], the related results can be derived.

Lemma 4.2. For any $f \in L^{2}(-1,1)$ and $\hat{\mathbf{z}}, \hat{\mathbf{x}} \in \mathbb{S}^{2}$, the following formulas hold.

$$
\begin{aligned}
& \int_{\mathbb{S}^{2}} f(\hat{\mathbf{z}} \cdot \hat{\mathbf{x}}) \operatorname{Re}\left(\mathrm{Y}_{n}^{m}(\hat{\mathbf{x}})\right) \mathrm{d} S(\hat{\mathbf{x}})=\lambda_{n} \operatorname{Re}\left(\mathrm{Y}_{n}^{m}(\hat{\mathbf{z}})\right), \\
& \int_{\mathbb{S}^{2}} f(\hat{\mathbf{z}} \cdot \hat{\mathbf{x}}) \operatorname{Im}\left(\mathrm{Y}_{n}^{m}(\hat{\mathbf{x}})\right) \mathrm{d} S(\hat{\mathbf{x}})=\lambda_{n} \operatorname{Im}\left(\mathrm{Y}_{n}^{m}(\hat{\mathbf{z}})\right),
\end{aligned}
$$

where $\lambda_{n}$ has been defined in (61).

Proof. See the Appendix.

Let us focus on the special variant of Lemma 4.1 and Lemma 4.2 for which $f(\hat{\mathbf{z}} \cdot \hat{\mathbf{x}})=\mathrm{e}^{-\mathrm{i} k_{0}|\mathbf{z}| \hat{\mathbf{z}} \cdot \hat{\mathbf{x}}}=$ $\mathrm{e}^{-\mathrm{i} k_{0} \mathbf{z} \cdot \hat{\mathbf{x}}}, \lambda_{n}$ is then given by

$$
\lambda_{n}=2 \pi \int_{-1}^{1} \mathrm{e}^{-\mathrm{i} k_{0}|\mathbf{z}| \hat{\mathbf{z}} \cdot \hat{\mathbf{x}}} \mathrm{P}_{n}(\hat{\mathbf{z}} \cdot \hat{\mathbf{x}}) \mathrm{d}(\hat{\mathbf{z}} \cdot \hat{\mathbf{x}}) .
$$

Since $\hat{\mathbf{z}} \cdot \hat{\mathbf{x}}=\cos \varphi$ where $\varphi$ is angle between two vectors $\hat{\mathbf{z}}$ and $\hat{\mathbf{x}}$, we have

$$
\begin{aligned}
\lambda_{n} & =2 \pi \int_{0}^{\pi} \mathrm{e}^{-\mathrm{i} k_{0}|\mathbf{z}| \cos \varphi} \mathrm{P}_{n}(\cos \varphi)(-\sin \varphi) \mathrm{d} \varphi \\
& =\frac{4 \pi}{\mathrm{i}^{n}} \mathrm{j}_{n}\left(k_{0}|\mathbf{z}|\right)
\end{aligned}
$$

thanks to the integral representation of the first kind spherical Bessel function $\mathrm{j}_{n}$ of order $n \in \mathbb{Z}$

$$
\mathrm{j}_{n}(\alpha)=\frac{(-\mathrm{i})^{n}}{2} \int_{0}^{\pi} \mathrm{e}^{\mathrm{i} \alpha \cos \varphi} \mathrm{P}_{n}(\cos \varphi) \sin \varphi \mathrm{d} \varphi .
$$

So, in our specific case, the results provided by the Lemmas 4.1 and 4.2 can be rewritten as

$$
\begin{aligned}
\int_{\mathbb{S}^{2}} \mathrm{e}^{-\mathrm{i} k_{0} \mathbf{z} \cdot \hat{\mathbf{x}}} \mathrm{Y}_{n}^{m}(\hat{\mathbf{x}}) \mathrm{d} S(\hat{\mathbf{x}}) & =\frac{4 \pi}{\mathrm{i}^{n}} \mathrm{j}_{n}\left(k_{0}|\mathbf{z}|\right) \mathrm{Y}_{n}^{m}(\hat{\mathbf{z}}), \\
\int_{\mathbb{S}^{2}} \mathrm{e}^{-\mathrm{i} k_{0} \mathbf{z} \cdot \hat{\mathbf{x}}} \operatorname{Re}\left(\mathrm{Y}_{n}^{m}(\hat{\mathbf{x}})\right) \mathrm{d} S(\hat{\mathbf{x}}) & =\frac{4 \pi}{\mathrm{i}^{n}} \mathrm{j}_{n}\left(k_{0}|\mathbf{z}|\right) \operatorname{Re}\left(\mathrm{Y}_{n}^{m}(\hat{\mathbf{z}})\right), \\
\int_{\mathbb{S}^{2}} \mathrm{e}^{-\mathrm{i} k_{0} \mathbf{z} \cdot \hat{\mathbf{x}}} \operatorname{Im}\left(\mathrm{Y}_{n}^{m}(\hat{\mathbf{x}})\right) \mathrm{d} S(\hat{\mathbf{x}}) & =\frac{4 \pi}{\mathrm{i}^{n}} \mathrm{j}_{n}\left(k_{0}|\mathbf{z}|\right) \operatorname{Im}\left(\mathrm{Y}_{n}^{m}(\hat{\mathbf{z}})\right),
\end{aligned}
$$

The next step is now to establish an analytical solution of (60) with the help of (67). 
Lemma 4.3. For $\mathbf{p} \in \mathbb{C}^{3}$ and $\mathbf{q} \in \mathbb{S}^{2}$, the following relation holds

$$
\int_{\mathbb{S}^{2}}\left(\underline{\mathbf{G}}_{\infty}\left(\hat{\mathbf{x}}, \mathbf{z}_{1}\right) \cdot \mathbf{p}\right) \cdot\left(\overline{\mathbf{G}_{\infty}\left(\hat{\mathbf{x}}, \mathbf{z}_{2}\right)} \cdot \mathbf{q}\right) \mathrm{d} S(\hat{\mathbf{x}})=\frac{8 \pi}{3}(\mathbf{p} \cdot \mathbf{q}) \mathrm{j}_{0}\left(k_{0}\left|\mathbf{z}_{1}-\mathbf{z}_{2}\right|\right)+\left(D_{1}+D_{2}\right) \mathbf{j}_{2}\left(k_{0}\left|\mathbf{z}_{1}-\mathbf{z}_{2}\right|\right),
$$

where $W_{1}$ and $W_{2}$ are given by

$$
W_{1}=-\frac{8 \pi}{3} \sqrt{\frac{\pi}{5}} \mathrm{Y}_{2}^{0}(\hat{\mathbf{z}})\left(p_{1} q_{1}+p_{2} q_{2}-2 p_{3} q_{3}\right)
$$

and

$$
\begin{aligned}
W_{2}=-8 \pi \sqrt{\frac{2 \pi}{15}}\left[\operatorname{Re}\left(\mathrm{Y}_{2}^{1}(\hat{\mathbf{z}})\right)\left(p_{1} q_{3}+p_{3} q_{1}\right)\right. & +\operatorname{Im}\left(\mathrm{Y}_{2}^{1}(\hat{\mathbf{z}})\right)\left(p_{2} q_{3}+p_{3} q_{2}\right) \\
& \left.-\operatorname{Re}\left(\mathrm{Y}_{2}^{2}(\hat{\mathbf{z}})\right)\left(p_{1} q_{1}-p_{2} q_{2}\right)-\operatorname{Im}\left(\mathrm{Y}_{2}^{2}(\hat{\mathbf{z}})\right)\left(p_{1} q_{2}+p_{2} q_{1}\right)\right]
\end{aligned}
$$

Here, $\hat{\mathbf{z}}=\left(\mathbf{z}_{1}-\mathbf{z}_{2}\right) /\left|\mathbf{z}_{1}-\mathbf{z}_{2}\right|$.

Proof. The introduction of the expression of the far-field approximation of dyadic green function (15) in (60) leads to

$$
\begin{aligned}
\int_{\mathbb{S}^{2}}\left(\underline{\mathbf{G}}_{\infty}\left(\hat{\mathbf{x}}, \mathbf{z}_{1}\right) \cdot \mathbf{p}\right) \cdot\left(\overline{\mathbf{G}_{\infty}\left(\hat{\mathbf{x}}, \mathbf{z}_{2}\right)} \cdot \mathbf{q}\right) \mathrm{d} S(\hat{\mathbf{x}}) & =\int_{\mathbb{S}^{2}}\left(\mathrm{e}^{-\mathrm{i} k_{0} \hat{\mathbf{x}} \cdot \mathbf{z}_{1}} \Delta(\hat{\mathbf{x}}) \cdot \mathbf{p}\right) \cdot\left(\mathrm{e}^{\mathrm{i} k_{0} \hat{\mathbf{x}} \cdot \mathbf{z}_{2}} \Delta(\hat{\mathbf{x}}) \cdot \mathbf{q}\right) \mathrm{d} S(\hat{\mathbf{x}}) \\
& =\int_{\mathbb{S}^{2}} \mathrm{e}^{-\mathrm{i} k_{0} \hat{\mathbf{x}} \cdot\left(\mathbf{z}_{1}-\mathbf{z}_{2}\right)} \mathbf{p} \cdot(\Delta(\hat{\mathbf{x}}) \cdot \mathbf{q}) \mathrm{d} S(\hat{\mathbf{x}}) \\
& \left.=\mathbf{p} \cdot \int_{\mathbb{S}^{2}}\left(\mathrm{e}^{-\mathrm{i} k_{0} \hat{\mathbf{x}} \cdot\left(\mathbf{z}_{1}-\mathbf{z}_{2}\right)} \Delta(\hat{\mathbf{x}}) \cdot \mathbf{q}\right)\right) \mathrm{d} S(\hat{\mathbf{x}})
\end{aligned}
$$

where the following properties $\Delta(\hat{\mathbf{x}})^{T}=\Delta(\hat{\mathbf{x}})$ and $\Delta(\hat{\mathbf{x}})^{2}=\Delta(\hat{\mathbf{x}})$ are used to go from $(71)$ to $(72)$, and the fact that $\mathbf{p}$ is a complex constant vector to go from (72) to (73).

The next step is now to establish an explicit form of $\Delta(\hat{\mathbf{x}}) \cdot \mathbf{q}$. The definition of $\Delta(\hat{\mathbf{x}})$ :

$$
\Delta(\hat{\mathbf{x}}):=\underline{\mathbf{I}}_{3}-\hat{\mathbf{x}} \otimes \hat{\mathbf{x}}=\left[\begin{array}{ccc}
1-\hat{x}_{1}^{2} & -\hat{x}_{1} \hat{x}_{2} & -\hat{x}_{1} \hat{x}_{3} \\
-\hat{x}_{1} \hat{x}_{2} & 1-\hat{x}_{2}^{2} & -\hat{x}_{2} \hat{x}_{3} \\
-\hat{x}_{1} \hat{x}_{3} & -\hat{x}_{1} \hat{x}_{2} & 1-\hat{x}_{3}^{2}
\end{array}\right]
$$

leads to

$$
(\Delta(\hat{\mathbf{x}}) \cdot \mathbf{q})_{s}=q_{s}-\hat{x}_{s}(\mathbf{q} \cdot \hat{\mathbf{x}}), s=1,2,3 .
$$

Using the expressions of the unit vector $\hat{\mathbf{x}}=(\sin \theta \cos \phi, \sin \theta \sin \phi, \cos \theta)^{T}$ and of the spherical harmonics $\mathrm{Y}_{n}^{m}$ as a function of the spherical coordinates:

$$
\begin{aligned}
& \mathrm{Y}_{2}^{0}(\theta, \phi)=\frac{1}{4} \sqrt{\frac{5}{\pi}}\left(3 \cos ^{2} \theta-1\right) \\
& \mathrm{Y}_{2}^{1}(\theta, \phi)=-\frac{1}{2} \sqrt{\frac{15}{2 \pi}} \sin \theta \cos \theta \mathrm{e}^{\mathrm{i} \phi} \\
& \mathrm{Y}_{2}^{2}(\theta, \phi)=\frac{1}{4} \sqrt{\frac{15}{2 \pi}} \sin ^{2} \theta \mathrm{e}^{2 \mathrm{i} \phi}
\end{aligned}
$$

we can express the first component of $\Delta(\hat{\mathbf{x}}) \cdot \mathbf{q}$ as

$$
\begin{aligned}
(\Delta(\hat{\mathbf{x}}) \cdot \mathbf{q})_{1} & =q_{1}-\sin \theta \cos \phi\left(q_{1} \sin \theta \cos \phi+q_{2} \sin \theta \sin \phi+q_{3} \cos \theta\right) \\
& =q_{1}-\frac{q_{1}}{2} \sin ^{2} \theta-\frac{q_{1}}{2} \sin ^{2} \theta \cos 2 \phi-\frac{q_{2}}{2} \sin ^{2} \theta \sin 2 \phi-q_{3} \sin \theta \cos \theta \cos \phi \\
& =\frac{2}{3} q_{1}+\frac{2}{3} q_{1} \sqrt{\frac{\pi}{5}} \mathrm{Y}_{2}^{0}-2 \sqrt{\frac{2 \pi}{15}}\left[q_{1} \operatorname{Re}\left(\mathrm{Y}_{2}^{2}(\hat{\mathbf{x}})\right)+q_{2} \operatorname{Im}\left(\mathrm{Y}_{2}^{2}(\hat{\mathbf{x}})\right)-q_{3} \operatorname{Re}\left(\mathrm{Y}_{2}^{1}(\hat{\mathbf{x}})\right)\right] .
\end{aligned}
$$

Similarly, the other components are obtained by

$$
\begin{aligned}
& (\Delta(\hat{\mathbf{x}}) \cdot \mathbf{q})_{2}=\frac{2}{3} q_{2}+\frac{2}{3} q_{2} \sqrt{\frac{\pi}{5}} \mathrm{Y}_{2}^{0}(\hat{\mathbf{x}})-2 \sqrt{\frac{2 \pi}{15}}\left[q_{1} \operatorname{Im}\left(\mathrm{Y}_{2}^{2}(\hat{\mathbf{x}})\right)-q_{2} \operatorname{Re}\left(\mathrm{Y}_{2}^{2}(\hat{\mathbf{x}})\right)-q_{3} \operatorname{Im}\left(\mathrm{Y}_{2}^{1}(\hat{\mathbf{x}})\right)\right] \\
& (\Delta(\hat{\mathbf{x}}) \cdot \mathbf{q})_{3}=\frac{2}{3} q_{3}-\frac{4}{3} q_{3} \sqrt{\frac{\pi}{5}} \mathrm{Y}_{2}^{0}(\hat{\mathbf{x}})+2 \sqrt{\frac{2 \pi}{15}}\left[q_{1} \operatorname{Re}\left(\mathrm{Y}_{2}^{1}(\hat{\mathbf{x}})\right)+q_{2} \operatorname{Im}\left(\mathrm{Y}_{2}^{1}(\hat{\mathbf{x}})\right)\right] .
\end{aligned}
$$


From the remarks $(67)$ and the fact that $Y_{0}^{0}(\hat{\mathbf{x}})=\frac{1}{\sqrt{4 \pi}}$, we have

$$
\int_{\mathbb{S}^{2}} \mathrm{e}^{-\mathrm{i} k \hat{\mathbf{x}} \cdot\left(\mathbf{z}_{1}-\mathbf{z}_{2}\right)} \mathrm{d} S(\hat{\mathbf{x}})=4 \pi \mathrm{j}_{0}\left(k\left|\mathbf{z}_{1}-\mathbf{z}_{2}\right|\right) .
$$

Combining either (79) or (80) with (67), for $\hat{\mathbf{z}}=\left(\mathbf{z}_{1}-\mathbf{z}_{2}\right) /\left|\mathbf{z}_{1}-\mathbf{z}_{2}\right|$, gives the following analytical expressions

$$
\begin{array}{r}
\int_{\mathbb{S}^{2}} \mathrm{e}^{-\mathrm{i} k_{0} \hat{\mathbf{x}} \cdot\left(\mathbf{z}_{1}-\mathbf{z}_{2}\right)}(\Delta(\hat{\mathbf{x}}) \cdot \mathbf{q})_{1} \mathrm{~d} S(\hat{\mathbf{x}})=\frac{8 \pi}{3} q_{1} \mathrm{j}_{0}\left(k_{0}\left|\mathbf{z}_{1}-\mathbf{z}_{2}\right|\right)-\frac{8 \pi}{3} q_{1} \sqrt{\frac{\pi}{5}} \mathrm{Y}_{2}^{0}(\hat{\mathbf{z}}) \mathrm{j}_{2}\left(k_{0}\left|\mathbf{z}_{1}-\mathbf{z}_{2}\right|\right) \\
+8 \pi \sqrt{\frac{2 \pi}{15}} \mathrm{j}_{2}\left(k_{0}\left|\mathbf{z}_{1}-\mathbf{z}_{2}\right|\right)\left[q_{1} \operatorname{Re}\left(\mathrm{Y}_{2}^{2}(\hat{\mathbf{z}})\right)+q_{2} \operatorname{Im}\left(\mathrm{Y}_{2}^{2}(\hat{\mathbf{z}})\right)-q_{3} \operatorname{Re}\left(\mathrm{Y}_{2}^{1}(\hat{\mathbf{z}})\right)\right] \\
\int_{\mathbb{S}^{2}} \mathrm{e}^{-\mathrm{i} k_{0} \hat{\mathbf{x}} \cdot\left(\mathbf{z}_{1}-\mathbf{z}_{2}\right)}(\Delta(\hat{\mathbf{x}}) \cdot \mathbf{q})_{2} \mathrm{~d} S(\hat{\mathbf{x}})=\frac{8 \pi}{3} q_{2} \mathrm{j}_{0}\left(k_{0}\left|\mathbf{z}_{1}-\mathbf{z}_{2}\right|\right)-\frac{8 \pi}{3} q_{2} \sqrt{\frac{\pi}{5}} \mathrm{Y}_{2}^{0}(\hat{\mathbf{z}}) \mathrm{j}_{2}\left(k_{0}\left|\mathbf{z}_{1}-\mathbf{z}_{2}\right|\right) \\
+8 \pi \sqrt{\frac{2 \pi}{15}} \mathrm{j}_{2}\left(k_{0}\left|\mathbf{z}_{1}-\mathbf{z}_{2}\right|\right)\left[q_{1} \operatorname{Im}\left(\mathrm{Y}_{2}^{2}(\hat{\mathbf{z}})\right)-q_{2} \operatorname{Re}\left(\mathrm{Y}_{2}^{2}(\hat{\mathbf{z}})\right)-q_{3} \operatorname{Im}\left(\mathrm{Y}_{2}^{1}(\hat{\mathbf{z}})\right)\right]
\end{array}
$$

and

$$
\begin{aligned}
\int_{\mathbb{S}^{2}} \mathrm{e}^{-\mathrm{i} k_{0} \hat{\mathbf{x}} \cdot\left(\mathbf{z}_{1}-\mathbf{z}_{2}\right)}(\Delta(\hat{\mathbf{x}}) \cdot \mathbf{q})_{3} \mathrm{~d} S(\hat{\mathbf{x}})=\frac{8 \pi}{3} & q_{3} \mathrm{j}_{0}\left(k_{0}\left|\mathbf{z}_{1}-\mathbf{z}_{2}\right|\right)+\frac{16 \pi}{3} q_{3} \sqrt{\frac{\pi}{5}} \mathrm{Y}_{2}^{0}(\hat{\mathbf{z}}) \mathrm{j}_{2}\left(k_{0}\left|\mathbf{z}_{1}-\mathbf{z}_{2}\right|\right) \\
& -8 \pi \sqrt{\frac{2 \pi}{15}} \mathrm{j}_{2}\left(k_{0}\left|\mathbf{z}_{1}-\mathbf{z}_{2}\right|\right)\left[q_{1} \operatorname{Re}\left(\mathrm{Y}_{2}^{1}(\hat{\mathbf{z}})\right)+q_{2} \operatorname{Im}\left(\mathrm{Y}_{2}^{1}(\hat{\mathbf{z}})\right)\right]
\end{aligned}
$$

They leads to

$$
\left.\int_{\mathbb{S}^{\not}} \mathbf{p} \cdot\left(\mathrm{e}^{-\mathrm{i} k_{0} \hat{\mathbf{x}} \cdot\left(\mathbf{z}_{1}-\mathbf{z}_{2}\right)} \Delta(\hat{\mathbf{x}}) \cdot \mathbf{q}\right)\right) \mathrm{d} S(\hat{\mathbf{x}})=\frac{8 \pi}{3}(\mathbf{p} \cdot \mathbf{q}) \mathrm{j}_{0}\left(k_{0}\left|\mathbf{z}_{1}-\mathbf{z}_{2}\right|\right)+\left(W_{1}+W_{2}\right) \mathrm{j}_{2}\left(k\left|\mathbf{z}_{1}-\mathbf{z}_{2}\right|\right),
$$

which complete the lemma. Here $C_{1}$ and $C_{2}$ are given by (69) and (70), respectively.

Now, under the small volume and spherical shape assumptions for the inhomogeneities, the DSM indicator function (58) is derived thanks to the Lemma 4.3 and asymptotic formula (21).

Theorem 4.4. Assume that the total number of observation directions $N$ is sufficiently large and each inhomogeneity is enough small $\left(\alpha_{m} \sqrt{\varepsilon_{m} / \varepsilon_{9}} \ll 0.5 \lambda\right)$. Then, the DSM indicator function has following representation formula.

$$
\mathcal{I}_{\mathrm{DSM}}^{\infty}(\mathbf{z} ; \hat{\mathbf{y}}, \hat{\mathbf{q}}) \approx \frac{\left|\mathcal{L}_{2}(\mathbf{z}, \hat{\mathbf{y}}, \hat{\mathbf{q}})\right|}{\max _{\mathbf{z} \in \Omega}\left|\mathcal{L}_{2}(\mathbf{z}, \hat{\mathbf{y}}, \hat{\mathbf{q}})\right|}
$$

where

$$
\mathcal{L}_{2}(\mathbf{z}, \hat{\mathbf{y}}, \hat{\mathbf{q}}):=\left|\sum_{m=1}^{M} A_{m} \mathrm{e}^{\mathrm{i} k_{0} \mathbf{r}_{m} \cdot \hat{\mathbf{y}}}\left[\frac{8 \pi}{3}\left(\left(\Delta(\hat{\mathbf{y}}) \cdot \hat{\mathbf{p}}^{t}\right) \cdot \hat{\mathbf{q}}\right) \mathrm{j}_{0}\left(k_{0}\left|\mathbf{r}_{m}-\mathbf{z}\right|\right)+\left(C_{3}+C_{4}\right) \mathrm{j}_{2}\left(k_{0}\left|\mathbf{r}_{m}-\mathbf{z}\right|\right)\right]\right|
$$

Here, for $\hat{\mathbf{z}}_{m}=\left(\mathbf{r}_{m}-\mathbf{z}\right) /\left|\mathbf{r}_{m}-\mathbf{z}\right|$

$$
C_{3}=-\frac{8 \pi}{3} \sqrt{\frac{\pi}{5}}\left[\left(\Delta(\hat{\mathbf{y}}) \cdot \hat{\mathbf{p}}^{t}\right)_{1} \hat{q}_{1}+\left(\Delta(\hat{\mathbf{y}}) \cdot \hat{\mathbf{p}}^{t}\right)_{2} \hat{q}_{2}-2\left(\Delta(\hat{\mathbf{y}}) \cdot \hat{\mathbf{p}}^{t}\right)_{3} \hat{q}_{3}\right] \mathrm{Y}_{2}^{0}\left(\hat{\mathbf{z}}_{m}\right)
$$

and

$$
\begin{aligned}
C_{4}= & 8 \pi \sqrt{\frac{2 \pi}{15}}\left[-\operatorname{Re}\left(\mathrm{Y}_{2}^{1}(\hat{\mathbf{z}})\right)\left\{\left(\Delta(\hat{\mathbf{y}}) \cdot \hat{\mathbf{p}}^{t}\right)_{1} \hat{q}_{3}+\left(\Delta(\hat{\mathbf{y}}) \cdot \hat{\mathbf{p}}^{t}\right)_{3} \hat{q}_{1}\right\}\right. \\
& -\operatorname{Im}\left(\mathrm{Y}_{2}^{1}(\hat{\mathbf{z}})\right)\left\{\left(\Delta(\hat{\mathbf{y}}) \cdot \hat{\mathbf{p}}^{t}\right)_{2} \hat{q}_{3}+\left(\Delta(\hat{\mathbf{y}}) \cdot \hat{\mathbf{p}}^{t}\right)_{3} \hat{q}_{2}\right\}+\operatorname{Re}\left(\mathrm{Y}_{2}^{2}(\hat{\mathbf{z}})\right)\left\{\left(\Delta(\hat{\mathbf{y}}) \cdot \hat{\mathbf{p}}^{t}\right)_{1} \hat{q}_{1}-\left(\Delta(\hat{\mathbf{y}}) \cdot \hat{\mathbf{p}}^{t}\right)_{2} \hat{q}_{2}\right\} \\
& \left.+\operatorname{Im}\left(\mathrm{Y}_{2}^{2}(\hat{\mathbf{z}})\right)\left\{\left(\Delta(\hat{\mathbf{y}}) \cdot \hat{\mathbf{p}}^{t}\right)_{1} \hat{q}_{2}+\left(\Delta(\hat{\mathbf{y}}) \cdot \hat{\mathbf{p}}^{t}\right)_{2} \hat{q}_{1}\right\}\right] .
\end{aligned}
$$


Proof. According to the the asymptotic formula of far-field pattern (19) and sufficiently large $N$,

$$
\begin{aligned}
\left|\left\langle\mathbf{E}_{\infty}(\hat{\mathbf{x}}, \hat{\mathbf{y}}), \underline{\mathbf{G}}_{\infty}(\mathbf{x}, \mathbf{z}) \hat{\mathbf{q}}\right\rangle_{L^{2}\left(\mathbb{S}^{2}\right)}\right| & \left.=\mid k_{0}^{2} \sum_{m=1}^{M} A_{m} \sum_{n=1}^{N} \underline{\mathbf{G}}_{\infty}\left(\mathbf{r}_{m}, \hat{\mathbf{x}}_{n}\right) \mathbf{E}^{i}\left(\mathbf{r}_{m}, \hat{\mathbf{y}}\right) \cdot \overline{\mathbf{G}_{\infty}(\mathbf{z}, \hat{\mathbf{x}}}\right) \hat{\mathbf{q}} \mid \\
& \approx\left|k_{0}^{2} \sum_{m=1}^{M} A_{m} \int_{\mathbb{S}^{2}} \underline{\mathbf{G}}_{\infty}\left(\mathbf{r}_{m}, \hat{\mathbf{x}}\right) \mathbf{E}^{i}\left(\mathbf{r}_{m}, \hat{\mathbf{y}}\right) \cdot \overline{\mathbf{G}_{\infty}(\mathbf{z}, \hat{\mathbf{x}})} \hat{\mathbf{q}} \mathrm{d} S(\hat{\mathbf{x}})\right| .
\end{aligned}
$$

By substituting $\hat{\mathbf{p}}^{t}=\mathbf{E}_{\infty}^{i}\left(\mathbf{r}_{m}, \hat{\mathbf{y}}\right)=\mathrm{i} \omega_{0} \mu_{0} \mathrm{e}^{\mathrm{i} k_{0} \mathbf{r}_{m} \cdot \hat{\mathbf{y}}} \Delta(\hat{\mathbf{y}}) \hat{\mathbf{p}}^{t}$ and $\mathbf{q}=\hat{\mathbf{q}}$ in Lemma 4.3,

$$
\begin{aligned}
\left|\left\langle\mathbf{E}_{\infty}(\hat{\mathbf{x}}, \hat{\mathbf{y}}), \underline{\mathbf{G}}_{\infty}(\mathbf{x}, \mathbf{z}) \hat{\mathbf{q}}\right\rangle_{L^{2}\left(\mathbb{S}^{2}\right)}\right| & \approx \mathrm{i} \omega_{0} \mu_{0} I l k_{0}^{2} \mid \sum_{m=1}^{M} A_{m} \mathrm{e}^{\mathrm{i} k_{0} \mathbf{r}_{m} \cdot \hat{\mathbf{y}}} \\
& \times\left[\frac{8 \pi}{3}\left(\left(\Delta(\hat{\mathbf{y}}) \hat{\mathbf{p}}^{t}\right) \cdot \hat{\mathbf{q}}\right) \mathrm{j}_{0}\left(k_{0}\left|\mathbf{r}_{m}-\mathbf{z}\right|\right)+\left(C_{3}+C_{4}\right) \mathrm{j}_{2}\left(k_{0}\left|\mathbf{r}_{m}-\mathbf{z}\right|\right)\right] \mid
\end{aligned}
$$

where $C_{3}$ and $C_{4}$ are given by (88) and (89), respectively. Thanks to the Hölder's inequality (44), the constant $i \omega_{0} \mu_{0} I l k_{0}^{2}$ is canceled and the proof is completed.

In the following, the structural properties of the DSM indicator function are discussed. Note that some remarks will be redundant due to the similarity between the formulations in the near-field and the far-field configurations.

Remark 4.1 (Properties of DSM with far-field data). According to Theorem 4.4, following properties of DSM indicator function in far-field configuration can be investigated.

1. As for Figure $11, \mathcal{I}_{\mathrm{DSM}}^{\infty}(\mathbf{z} ; \hat{\mathbf{q}})$ has a maximum of magnitude at $\mathbf{z}=\mathbf{r}_{m} \in \tau_{m}$ since $\mathrm{j}_{0}\left(k_{0}\left|\mathbf{r}_{m}-\mathbf{z}\right|\right)=1$ and $\mathrm{j}_{2}\left(k_{0}\left|\mathbf{r}_{m}-\mathbf{z}\right|\right)=0$ in that case. However, unexpected artifacts can be found in the maps of $\mathcal{I}_{\mathrm{DSM}}^{\infty}(\mathbf{z} ; \hat{\mathbf{q}})$ due to $\mathrm{j}_{2}\left(k_{0}\left|\mathbf{r}_{m}-\mathbf{z}\right|\right)>0$ if $\mathbf{z} \notin \tau_{m}$.

2. According to the facts that stated in Remark 3.12 , when dealing with multiple defects having different physical properties (e.g., size and/or permittivity, etc.) in the medium, the one having a smaller size or permittivity than the others will difficult to be identified through the maps of $\mathcal{I}_{\mathrm{DSM}}^{\infty}(\mathbf{z} ; \hat{\mathbf{q}})$.

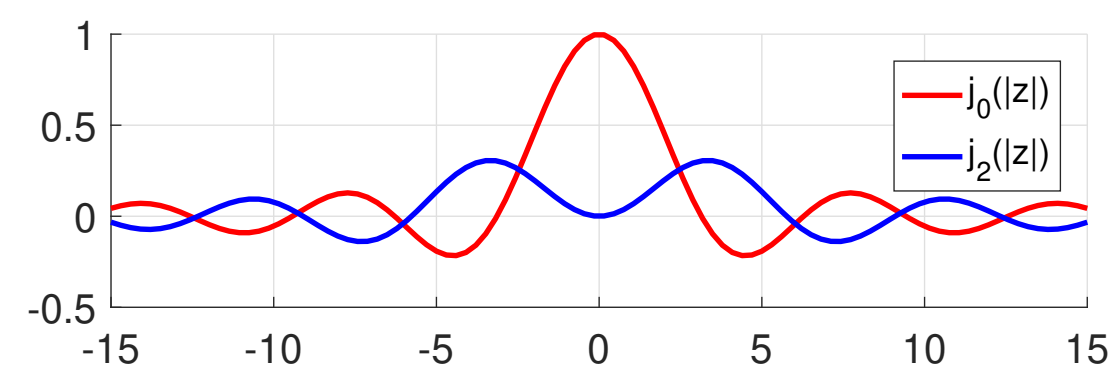

Figure 11: One-dimensional plot of the spherical Bessel function with orders 0, 2

Now, we investigate the method to choose proper polarization of test dipole based on Theorem 4.4 as the following remark.

Remark 4.2 (Investigation of choosing proper $\hat{\mathbf{q}}$ ). Since $\mathrm{j}_{0}\left(k_{0}\left|\mathbf{r}_{m}-\mathbf{z}\right|\right)$ contributes to retrieve the information about inhomogeneities in the map of $\mathcal{I}_{\mathrm{DSM}}^{\infty}(\mathbf{z} ; \hat{\mathbf{q}})$, it is necessary to choose the polarization $\hat{\mathbf{q}}$ at least satisfying following condition:

$$
\left(\Delta(\hat{\mathbf{y}}) \cdot \hat{\mathbf{p}}^{t}\right) \cdot \hat{\mathbf{q}} \neq 0 \quad \text { if and only if } \quad \hat{\mathbf{p}}^{t} \cdot \hat{\mathbf{q}} \neq(\hat{\mathbf{y}} \cdot \hat{\mathbf{q}})\left(\hat{\mathbf{y}} \cdot \hat{\mathbf{p}}^{t}\right)
$$

which is similar to Remark 3.2, but, on the contrary to (45), the condition (92) does not involve information about the localization of the defect. 
1. Nevertheless, it is impossible to get an analytic solution of $\hat{\mathbf{q}}$ without a prior information about $\tau_{m}$ because the latter is essential to eliminate the disturbing terms $\mathrm{j}_{2}\left(k_{0}\left|\mathbf{r}_{m}-\mathbf{z}\right|\right)$ by making $C_{1}+C_{2}=0$. Hence, we can again obtain the proper polarization of test dipole with the same process in the nearfield case proposed in (46) by

$$
\mathcal{I}_{\mathrm{DSMP}}^{\infty}(\mathbf{z} ; \mathbf{y})=\max _{\hat{\mathbf{q}} \in \mathbb{S}^{2}}\left\{\mathcal{I}_{\mathrm{DSM}}^{\infty}(\mathbf{z} ; \mathbf{y}, \hat{\mathbf{q}})\right\}
$$

2. Similar to the near-field case in Corollary 3.2, to select $\hat{\mathbf{q}}$ as $\hat{\mathbf{p}}^{t}$ will be one of proper way to image the target via $\mathcal{I}_{\mathrm{DSM}}^{\infty}(\mathbf{z} ; \hat{\mathbf{q}})$. Because if $\hat{\mathbf{q}}=\hat{\mathbf{p}}^{t}$,

$$
\hat{\mathbf{p}}^{t} \cdot \hat{\mathbf{q}}-(\hat{\mathbf{y}} \cdot \hat{\mathbf{q}})\left(\hat{\mathbf{y}} \cdot \hat{\mathbf{p}}^{t}\right)=1-\cos ^{2} \vartheta
$$

where $\vartheta$ is between angle of $\hat{\mathbf{p}}^{t}$ and $\hat{\mathbf{y}}$. Since $\hat{\mathbf{p}}^{t} \nVdash \hat{\mathbf{q}}$, the necessary condition (92) holds.

\subsection{Numerical simulations with far-field data}

Our analysis and proposal are verified through the numerical simulations using synthetic far-field data obtained by FEKO to which a $20 \mathrm{~dB}$ white Gaussian random noise using the MATLAB function awgn is added. The configurations are the same as Example 3.1 and Example 3.2 in section 3.2 except but in the far-field configuration. In this simulations, we again consider evenly distributed $\hat{\mathbf{q}}_{t} \in \mathbb{S}^{2}, t=1,2, \cdots, 201$ to apply our proposal DSMP.

The spherical coordinate system is again used to present the information about direction and polarization of source and observation. A single incident impinging wave of direction $\left(-1,90^{\circ}, 0^{\circ}\right)$ and polarization $\hat{\mathbf{p}}^{t}=\left(1,0^{\circ}, 0^{\circ}\right)(=(0,0,1)$ in Cartesian coordinate system $)$ is considered. The observation directions are evenly distributed with $\theta^{r}$ varying from $0^{\circ}$ to $350^{\circ}$ with $10^{\circ}$-step and $\phi^{r}$ varying from $10^{\circ}$ to $170^{\circ}$ with $10^{\circ}$-step. Since the far-field configuration is considered, only the $\theta^{r}$ - and $\phi^{r}$-components of the far-field pattern are obtained. Then, a transformed far-field pattern $\widetilde{\mathbf{E}}_{\infty}(\mathbf{x}, \mathbf{y})=\underline{\mathbf{P E}}_{\infty}(\mathbf{x}, \mathbf{y})$ is used to adjust our theoretical approach (using three components of scattered field along $x, y$ and $z$ ) where the transition matrix is defined by $\underline{\mathbf{P}}=\left[\hat{r}^{r} ; \hat{\theta}^{r} ; \hat{\phi}^{r}\right] \in \mathbb{R}^{3 \times 3}$ with

$$
\begin{aligned}
& \hat{r}^{r}=\left(\sin \theta^{r} \cos \phi^{r}, \sin \theta^{r} \sin \phi^{r}, \cos \theta^{r}\right)^{T}, \\
& \hat{\theta}^{r}=\left(\cos \theta^{r} \cos \phi^{r}, \cos \theta^{r} \sin \phi^{r}, \cos \theta\right)^{T}, \\
& \hat{\phi}^{r}=\left(-\sin \phi^{r}, \cos \phi^{r}, 0\right)^{T},
\end{aligned}
$$

in Cartesian coordinate system. As in section 3.2, the results are visualized using volume slice plane and isosurface using isosurface parameter $\nu$ given in (54). The accuracy of the methods is measured by the Jaccard index in (55)

Example 4.1 (Single small sphere). Let us remind the information about the inhomogeneity $\tau$ with $\mathbf{r}=(0.1 \mathrm{~m}, 0.1 \mathrm{~m}, 0.1 \mathrm{~m}), \alpha=0.1 \lambda=0.04 \mathrm{~m}$, and $\varepsilon=5 \varepsilon_{0}$, respectively.

As shown in Figures 12, 13, and 14, the results are similar to the ones obtained within the nearfield configuration (Example 3.1). The DSM results are getting worse as the direction $\hat{\mathbf{q}}$ is changed in $0^{\circ} \leq \theta \leq 70^{\circ}$ for fixed $\phi=45^{\circ}$. Whereas, the inhomogeneity is correctly identified via DSM with $\hat{\mathbf{p}}^{t}$ or DSMP. Hence, we can validate that both DSM with $\hat{\mathbf{q}}=\hat{\mathbf{p}}^{t}$ and DSMP would be a good choice for imaging of the inhomogeneity, but our proposal DSMP is no need to use the information about polarization of incident field even with more computational cost. Furthermore, we can verify that the accuracy of DSMP is slightly better than the traditional DSM by the Jaccard index in Figure 15.

Example 4.2 (Two small spheres with same radii and different permittivity). Similar to example 3.2, two small spherical dielectric inhomogeneities with same radii $\alpha_{m}=0.1 \lambda=0.04 \mathrm{~m}$ but different permittivity $\varepsilon_{1}=3 \varepsilon_{0}, \varepsilon_{2}=5 \varepsilon_{0}$ are considered. They are located at $\mathbf{r}_{1}=(0.1 \mathrm{~m}, 0.1 \mathrm{~m}, 0.1 \mathrm{~m})$ and $\mathbf{r}_{2}=$ $(-0.2 \mathrm{~m},-0.1 \mathrm{~m},-0.1 \mathrm{~m})$, respectively.

Figure 16 shows that the issue of choosing the proper polarization of test dipole still occurs as the previous example. DSM with the polarization as $\hat{\mathbf{p}}^{t}$ (Figure 17) or our proposal DSMP (Figure 18) handle it as expected. However, as stated in Remark 4.1, the inhomogeneity having the smaller permittivity is difficult to be identified with both DSM and DSMP, which is similar to the different radii case in example 3.2. The Figure 19 shows that the imaging performance is not enough good to identify all inhomogeneities via both DSM with $\hat{\mathbf{q}}=\hat{\mathbf{p}}^{t}$ and DSMP. 
$\theta=0^{\circ}$ and $\phi=45^{\circ}$
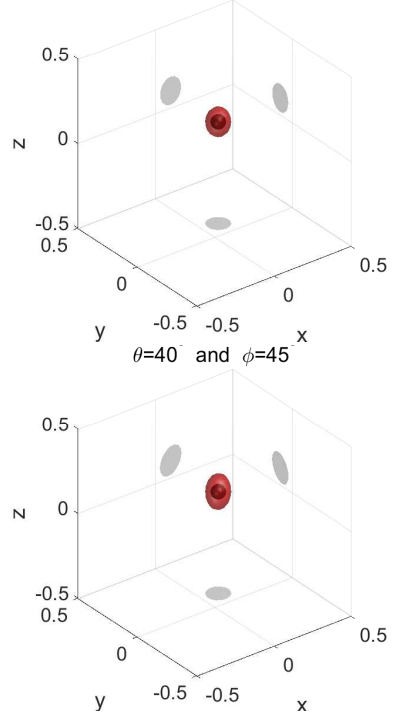

$\theta=10^{\circ}$ and $\phi=45$
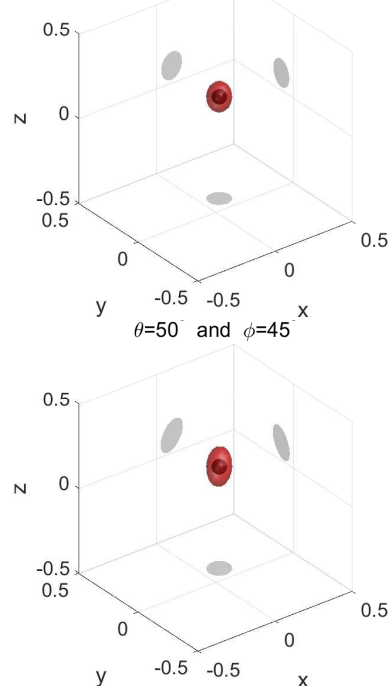

$\theta=20^{\circ}$ and $\phi=45$
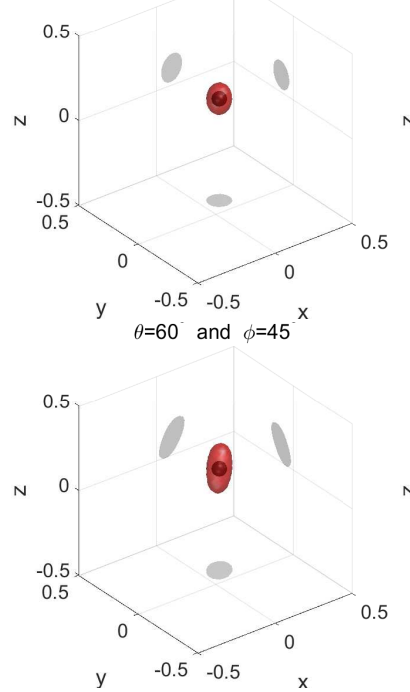

$\theta=30^{\circ}$ and $\phi=45$
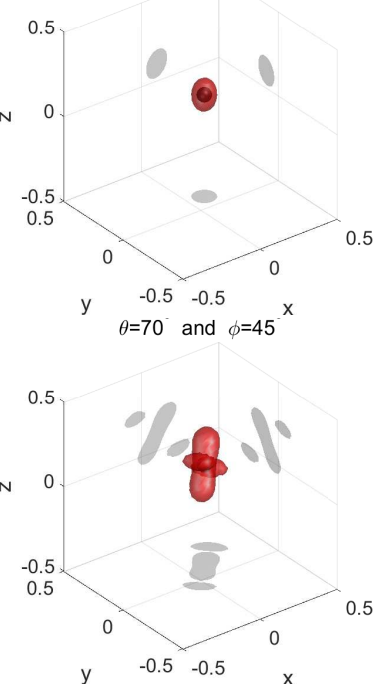

Figure 12: (Example 4.1) Maps of $\mathcal{I}_{\mathrm{DSM}}(\mathbf{z} ; \mathbf{y}, \hat{\mathbf{q}})$ using isosurface with $\rho=0.8$, where $\hat{\mathbf{q}}=(1, \theta, \phi)$

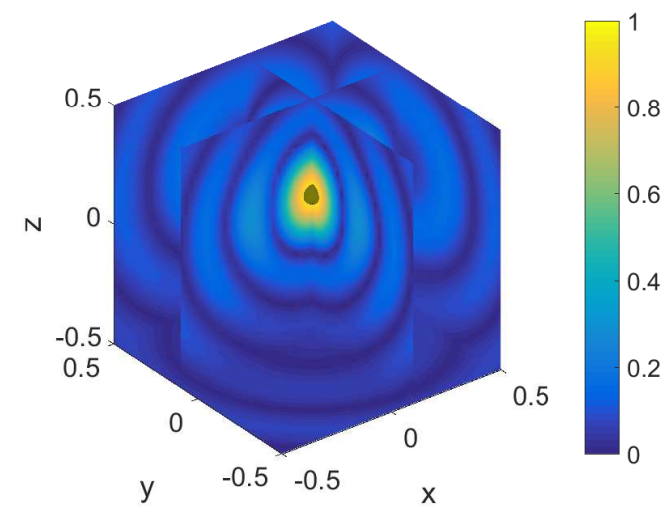

(a) Volume slice with $\mathbf{x}=0.1$ and $\mathbf{y}=0.1$ planes

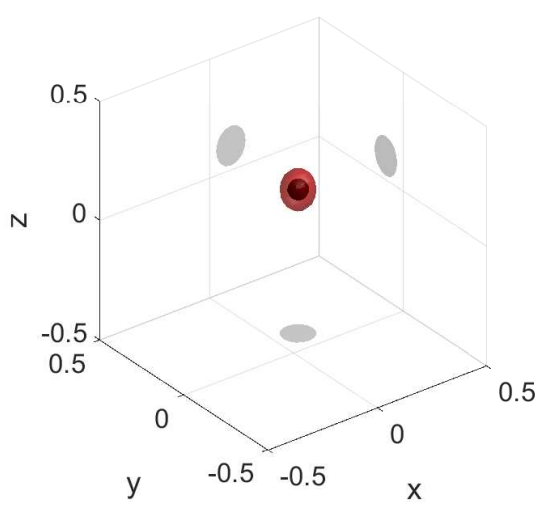

(b) Isosurface with $\rho=0.6$

Figure 13: (Example 4.1) Maps of $\mathcal{I}_{\mathrm{DSM}}(\mathbf{z} ; \mathbf{y}, \hat{\mathbf{q}})$, where $\hat{\mathbf{q}}=\left(1,0^{\circ}, 45^{\circ}\right)=(0,0,1)=\hat{\mathbf{p}}^{t}$

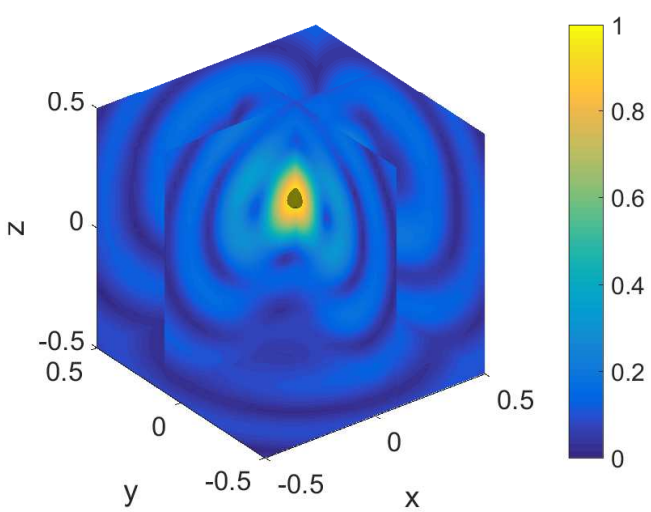

(a) Volume slice with $\mathbf{x}=0.1$ and $\mathbf{y}=0.1$ planes

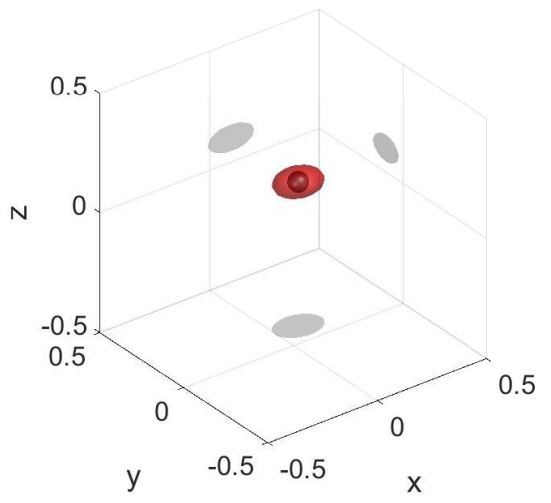

(b) Isosurface with $\rho=0.6$

Figure 14: (Example 4.1) Maps of $\mathcal{I}_{\mathrm{DSMP}}(\mathbf{z} ; \mathbf{y})$

\section{Illustration with experimental far-field data}

Additional results obtained using experimental data are presented thanks to Fresnel experimental data (http://www.fresnel.fr/3Ddatabase/index.php), the details of which being found in [42]. The fre- 


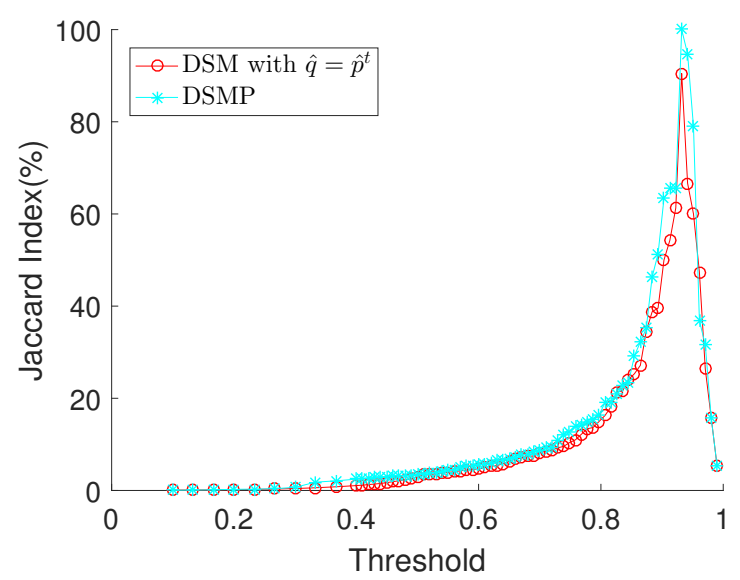

Figure 15: (Example 4.1) Jaccard index of $\mathcal{I}_{\mathrm{DSM}}^{\infty}\left(\mathbf{z} ; \mathbf{y}, \hat{\mathbf{q}}=\hat{\mathbf{p}}^{t}\right)$ and $\mathcal{I}_{\mathrm{DSMP}}^{\infty}(\mathbf{z} ; \mathbf{y})$
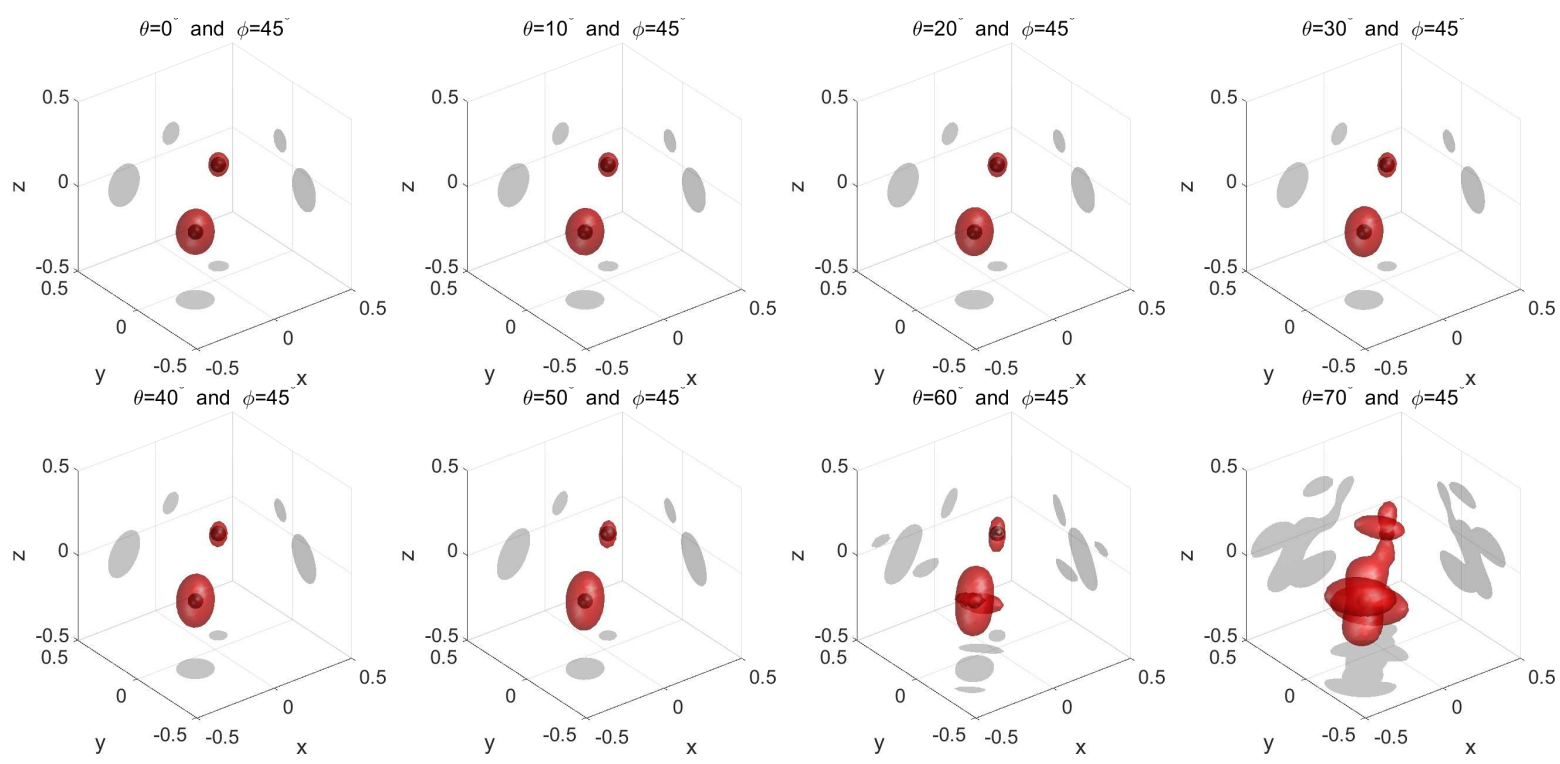

Figure 16: (Example 4.2) Maps of $\mathcal{I}_{\mathrm{DSM}}(\mathbf{z} ; \mathbf{y}, \hat{\mathbf{q}})$ using isosurface with $\rho=0.5$, where $\hat{\mathbf{q}}=(1, \theta, \phi)$

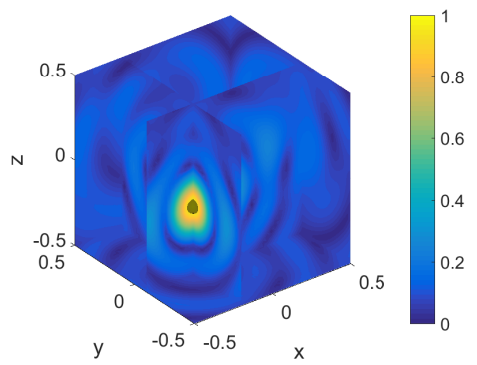

(a) Volume slice with $\mathbf{x}=0.1$ and $\mathbf{y}=(\mathrm{b})$ Volume slice with $\mathbf{x}=-0.2$ and 0.1 planes

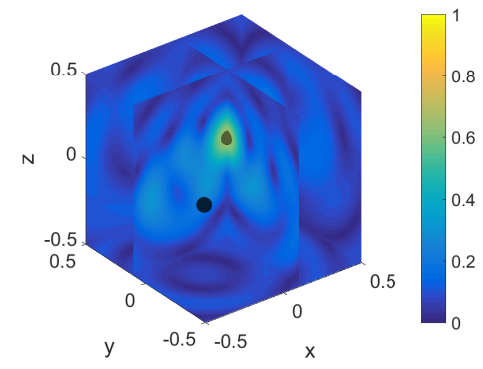

$\mathbf{y}=-0.1$ planes

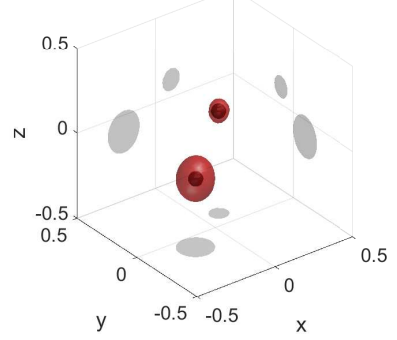

(c) Isosurface with $\rho=0.6$

Figure 17: (Example 4.2) Maps of $\mathcal{I}_{\mathrm{DSM}}(\mathbf{z} ; \mathbf{y}, \hat{\mathbf{q}})$, where $\hat{\mathbf{q}}=\left(1,0^{\circ}, 45^{\circ}\right)=\hat{\mathbf{p}}^{t}$

quency is chosen as $f_{0}=3 \mathrm{GHz}$ to be close to the our theoretical condition, the corresponding wavelength being then $\lambda_{0} \approx 0.1 \mathrm{~m}$. Among a various types of defects, two small dielectric sphere of same radii $\alpha_{m} \approx 0.25 \lambda_{0}$, permittivity $\varepsilon_{m}=2.6 \varepsilon_{0}$ with $\mathbf{r}_{1}=\left(0.25 \lambda_{0}, 0,0\right)$ and $\mathbf{r}_{2}=\left(-0.25 \lambda_{0}, 0,0\right)$ is selected (experimental file name is TwoSpheres_PP.exp and TwoSpheres_TP.exp). The region of interest $\Omega$ is a cube of $2.5 \lambda_{0}$ side length evenly discretized in $41 \times 41 \times 41$ voxels. The DSMP is obtained by considering 


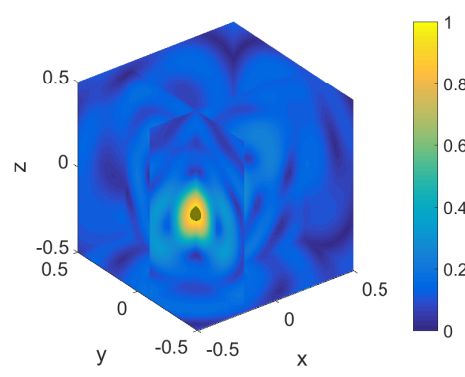

(a) Volume slice with $\mathbf{x}=0.1$ and $\mathbf{y}=(\mathrm{b})$ Volume slice with $\mathbf{x}=-0.2$ and
0.1 planes
$\mathbf{y}=-0.1$ planes 0.1 planes

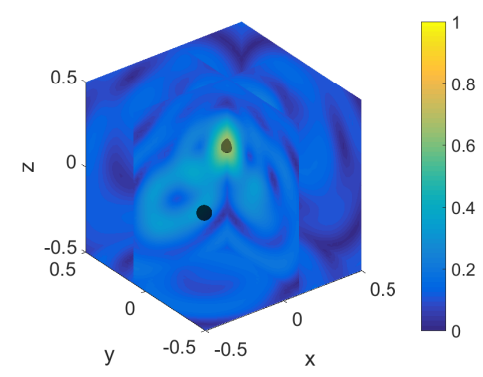

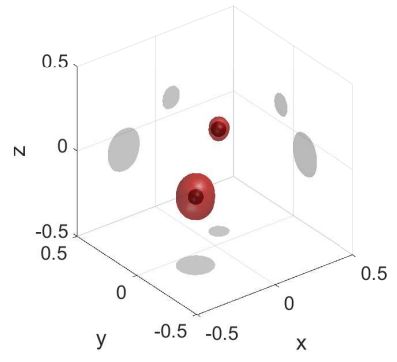

(c) Isosurface with $\rho=0.6$

Figure 18: (Example 4.2) Maps of $\mathcal{I}_{\mathrm{DSMP}}(\mathbf{z} ; \mathbf{y})$

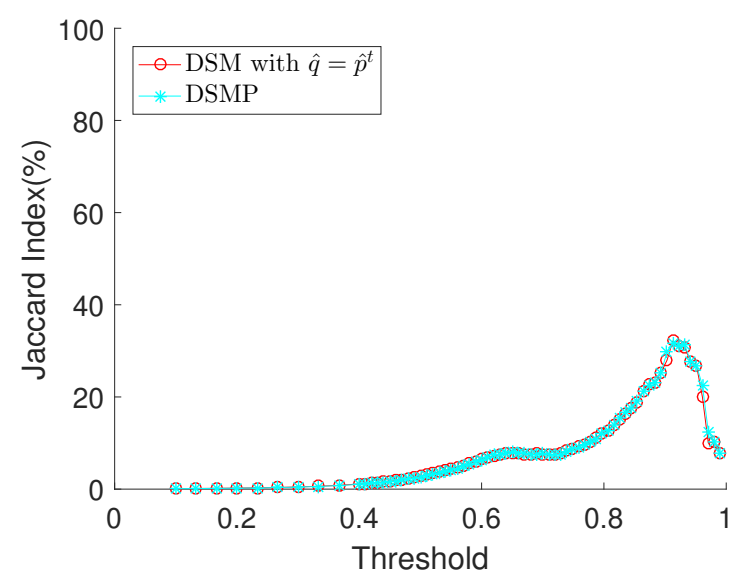

Figure 19: (Example 4.2) Jaccard index of $\mathcal{I}_{\mathrm{DSM}}^{\infty}\left(\mathbf{z} ; \mathbf{y}, \hat{\mathbf{q}}=\hat{\mathbf{p}}^{t}\right)$ and $\mathcal{I}_{\mathrm{DSMP}}^{\infty}(\mathbf{z} ; \mathbf{y})$

$\hat{\mathbf{q}}_{t} \in \mathbb{S}^{2}, t=1,2, \cdots, 201$.

It is worth note that

- Since the locations of receivers and transmitters are far from the center $\left(1.796 \mathrm{~m} \approx 18 \lambda_{0}\right)$ in the experimental configuration, the scattered electric field can be regarded as the far-field pattern.

- Due to the limitation of the experimental setup, only $N=36$ observation directions are considered, but many $L=81$ incident fields can be applied. However, thanks to the reciprocity of the roles of the source and the receiver can be exchanged [43].

- To adjust our theoretical approach, the transformed scattered field $\widetilde{\mathbf{E}}_{\infty}(\mathbf{x}, \mathbf{y})=\underline{\mathbf{P}}_{\infty}(\mathbf{x}, \mathbf{y})$ is used for imaging. Here $\underline{\mathbf{P}}=\left[\hat{r}^{r} ; \hat{\theta}^{r} ; \hat{\phi}^{r}\right] \in \mathbb{R}^{3 \times 3}$ is the transition matrix given by (95).

$N=81$ directions of observation are evenly distributed in polar angle $\theta^{r}$ from $30^{\circ}$ to $150^{\circ}$ with a $15^{\circ}$ step and in azimuthal angle $\phi^{r}$ from $0^{\circ}$ to $320^{\circ}$ with a $40^{\circ}$ step. The two tangential components (along with $\theta^{r}$ and $\phi^{r}$ ) of the scattered field are measured. Due to the design of experimental system, $L=27$ incident plane wave polarized along $\theta^{t}$ and located only on the azimuthal plane have been recorded, the impinging directions are defined as $\theta^{t}=90^{\circ}$ and $\phi^{t}$ varying from $50^{\circ}$ to $310^{\circ}$ with a $10^{\circ}$ step. Among those $L$ incidences only the one characterized by $\theta=90^{\circ}$ and $\phi=50^{\circ}$ has been used for the reconstruction of the inhomogeneities.

The results are presented Figures 20, 21, and 22. The choice of the test dipole as $\hat{\mathbf{q}}=\hat{\mathbf{p}}^{t}$ provides excellent results, but a little unexpected artifact is found. But we can eliminate it have to get better results to retrieve the location of the inhomogeneities with only a single impinging direction. In other words, the DSMP has a few better accuracy than tradition DSM in experimental data case while they have almost the same efficiency in synthetic data case. The Jaccard index in Figure 23 can confirm this observation. 

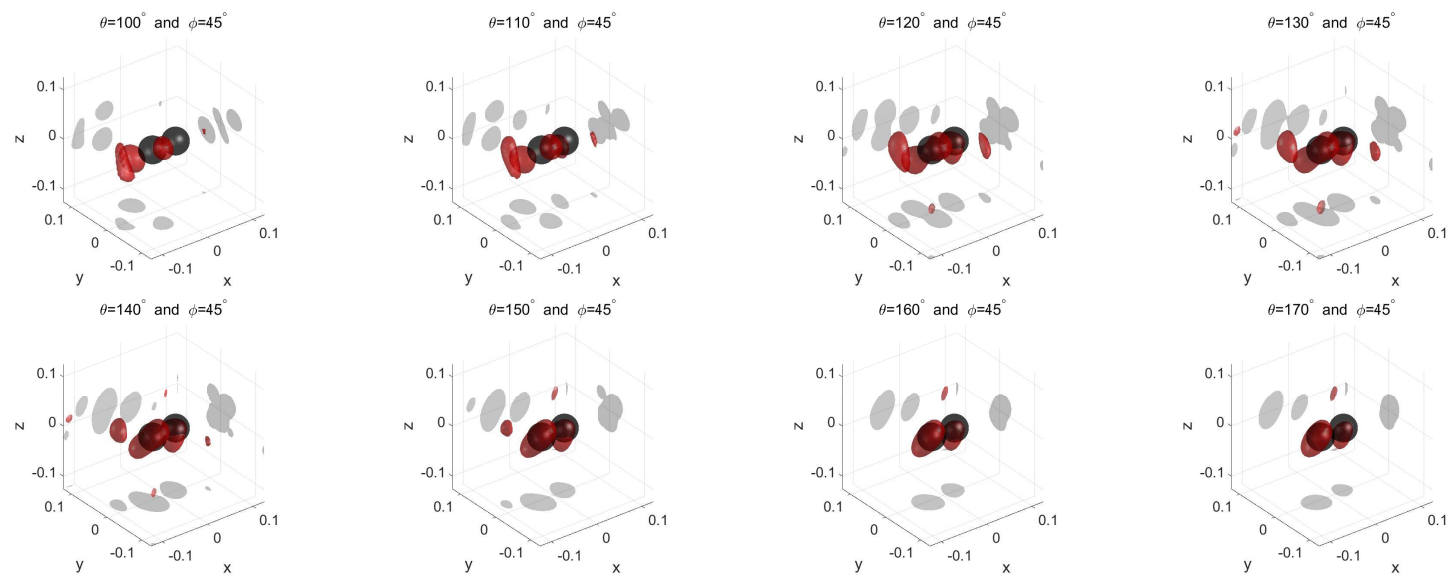

Figure 20: Maps of $\mathcal{I}_{\mathrm{DSM}}^{\infty}(\mathbf{z} ; \mathbf{y}, \hat{\mathbf{q}})$ using isosurface with $\rho=0.8$, where $\hat{\mathbf{q}}=(1, \theta, \phi)$

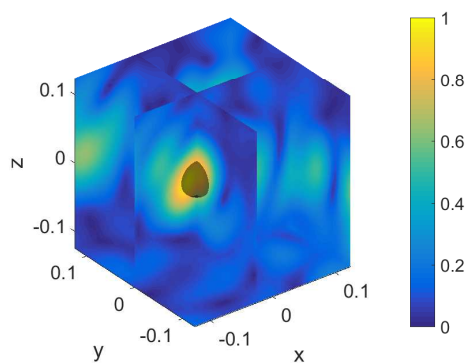

(a) Volume slice with $\mathbf{x}=-0.2$ and $\mathbf{y}=$ (b) Volume slice with $\mathbf{x}=0.1$ and $\mathbf{y}=$ -0.1 planes

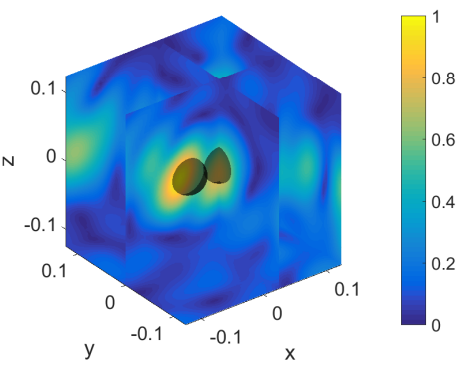

0.1 planes

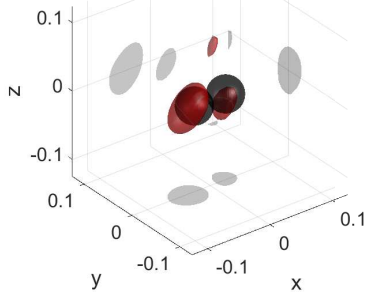

(c) Isosurface with $\rho=0.7$

Figure 21: Maps of $\mathcal{I}_{\mathrm{DSM}}^{\infty}(\mathbf{z} ; \mathbf{y}, \hat{\mathbf{q}})$, where $\hat{\mathbf{q}}=\left(1,180^{\circ}, 45^{\circ}\right)=\hat{\mathbf{p}}^{t}$

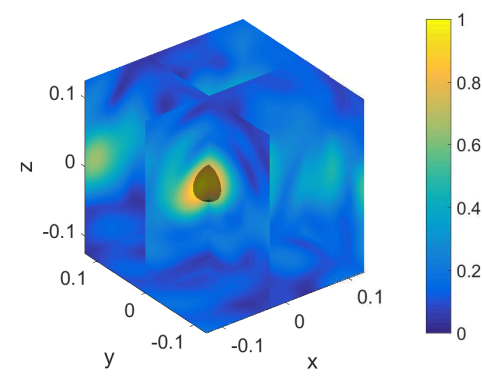

(a) Volume slice with $\mathbf{x}=-0.2$ and $\mathbf{y}=(\mathrm{b})$ Volume slice with $\mathbf{x}=0.1$ and $\mathbf{y}=$ -0.1 planes

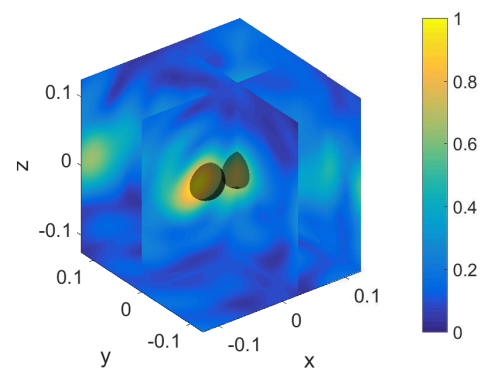

0.1 planes

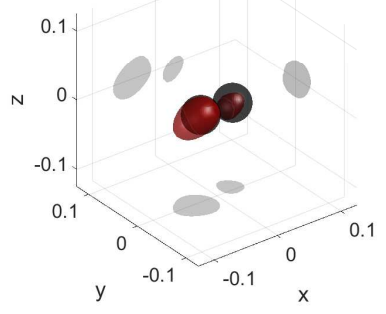

(c) Isosurface with $\rho=0.7$

Figure 22: Maps of $\mathcal{I}_{\mathrm{DSMP}}^{\infty}(\mathbf{z} ; \mathbf{y})$

\section{Conclusion}

In this article, the analytical expressions of the DSM indicator function within the small obstacle hypothesis are provided in both near-field and far-field configuration thanks to the asymptotic formula of the scattered field. With such an analysis, we explain the reasons for which DSM can localize the targets and for which some artifacts might appear. Moreover, the relation of the choice of the test dipole polarization and the efficiency of DSM is exhibited, and some methods to provide a better option is proposed with a theoretical validation. The approach is validated using synthetic data and experimental data when available.

Throughout this work, we only consider the full-view configuration. However, it might be impossible to obtain data in such a setting in real applications. Hence, the analysis of DSM in restricted configurations such as mono-static and limited-view configurations is of interest. 


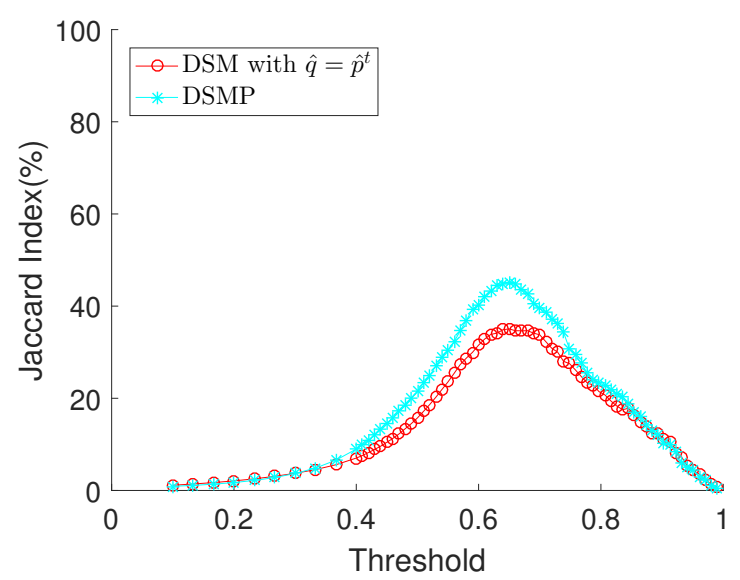

Figure 23: Jaccard index of $\mathcal{I}_{\mathrm{DSM}}^{\infty}\left(\mathbf{z} ; \mathbf{y}, \hat{\mathbf{q}}=\hat{\mathbf{p}}^{t}\right)$ and $\mathcal{I}_{\mathrm{DSMP}}^{\infty}(\mathbf{z} ; \mathbf{y})$

\section{Acknowledgment}

The authors are very grateful to Dominique Lesselier and Won-Kwang Park for their valuable advice.

\section{Appendix}

\section{Proof of Lemma 4.2}

For fixed $\hat{\mathbf{z}}$, choose an orthogonal matrix $A$ depending on $\hat{\mathbf{z}}$ such that $A^{-1} \hat{\mathbf{z}}=A^{T} \hat{\mathbf{z}}=\hat{\mathbf{z}}^{\prime}=(0,0,1)^{T}$, i.e., $\hat{\mathbf{z}}^{\prime}$ is "north pole". Then the transformation $\hat{\mathbf{x}}=A \hat{\mathbf{x}}^{\prime}$ leads that

$$
\int_{\mathbb{S}^{2}} f(\hat{\mathbf{z}} \cdot \hat{\mathbf{x}}) \mathrm{Y}_{n}^{m}(\hat{\mathbf{x}}) \mathrm{d} S(\hat{\mathbf{x}})=\int_{\mathbb{S}^{2}} f\left(\hat{\mathbf{z}} \cdot A \hat{\mathbf{x}}^{\prime}\right) \mathrm{Y}_{n}^{m}\left(A \hat{\mathbf{x}}^{\prime}\right) \mathrm{d} S\left(\hat{\mathbf{x}}^{\prime}\right)=\int_{\mathbb{S}^{2}} f\left(\hat{\mathbf{z}}^{\prime} \cdot \hat{\mathbf{x}}\right) \mathrm{Y}_{n}^{m}\left(A \hat{\mathbf{x}}^{\prime}\right) \mathrm{d} S(\hat{\mathbf{x}})
$$

Because the orthonormal matrix $A$ is isometry, the function $\mathrm{Y}_{n}^{m}(A \hat{\mathbf{x}})$ is again a spherical harmonic of order $n$ and it can be expressed as

$$
\mathrm{Y}_{n}^{m}(A \hat{\mathbf{x}})=\sum_{k=-n}^{n} a_{k} \mathrm{Y}_{n}^{k}(\hat{\mathbf{x}}) \text { where } a_{k}=\int_{\mathbb{S}^{2}} \mathrm{Y}_{n}^{m}(A \hat{\mathbf{x}}) \mathrm{Y}_{n}^{-k}(\hat{\mathbf{x}}) \mathrm{d} S(\hat{\mathbf{x}})
$$

We know that

$$
\begin{aligned}
& \operatorname{Re}\left(\mathrm{Y}_{n}^{m}(A \hat{\mathbf{x}})\right)=\sum_{k=-n}^{n} \operatorname{Re}\left(a_{k}\right) \operatorname{Re}\left(\mathrm{Y}_{n}^{k}(\hat{\mathbf{x}})\right)-\operatorname{Im}\left(a_{k}\right) \operatorname{Im}\left(\mathrm{Y}_{n}^{k}(\hat{\mathbf{x}})\right) \\
& \operatorname{Im}\left(\mathrm{Y}_{n}^{m}(A \hat{\mathbf{x}})\right)=\sum_{k=-n}^{n} \operatorname{Re}\left(a_{k}\right) \operatorname{Im}\left(\mathrm{Y}_{n}^{k}(\hat{\mathbf{x}})\right)+\operatorname{Im}\left(a_{k}\right) \operatorname{Re}\left(\mathrm{Y}_{n}^{k}(\hat{\mathbf{x}})\right) .
\end{aligned}
$$

By introducing (98) in (96) and by expressing $\hat{\mathbf{x}}$ in spherical coordinates $\hat{\mathbf{x}}=(\sin \theta \cos \phi, \sin \theta \sin \phi, \cos \theta)^{T}$ (note that $\hat{\mathbf{z}}^{\prime} \cdot \hat{\mathbf{x}}=\cos \theta$ ), the following expressions are obtained

$$
\begin{aligned}
& \int_{\mathbb{S}^{2}} f(\hat{\mathbf{z}} \cdot \hat{\mathbf{x}}) \operatorname{Re}\left(\mathrm{Y}_{n}^{m}(\hat{\mathbf{x}})\right) \mathrm{d} S(\hat{\mathbf{x}}) \\
& =\sum_{k=-n}^{n} \int_{\mathbb{S}^{2}} f\left(\hat{\mathbf{z}}^{\prime} \cdot \hat{\mathbf{x}}\right)\left[\operatorname{Re}\left(a_{k}\right) \operatorname{Re}\left(\mathrm{Y}_{n}^{k}(\hat{\mathbf{x}})\right)-\operatorname{Im}\left(a_{k}\right) \operatorname{Im}\left(\mathrm{Y}_{n}^{k}(\hat{\mathbf{x}})\right)\right] \mathrm{d} S(\hat{\mathbf{x}}) \\
& =\sum_{k=-n}^{n} \sqrt{\frac{(2 n+1)(n-|k|) !}{4 \pi(n+|k| !)}} \int_{0}^{\pi} \int_{0}^{2 \pi} f(\cos \theta) P_{n}^{|k|}(\cos \theta)\left[\operatorname{Re}\left(a_{k}\right) \cos (k \phi)-\operatorname{Im}\left(a_{k}\right) \sin (k \phi)\right] \mathrm{d} \phi \sin \theta \mathrm{d} \theta \\
& =\operatorname{Re}\left(a_{0}\right) \sqrt{\frac{2 n+1}{4 \pi}}\left(2 \pi \int_{0}^{\pi} f(\cos \theta) P_{n}(\cos \theta) \sin \theta \mathrm{d} \theta\right) .
\end{aligned}
$$


and

$$
\begin{aligned}
& \int_{\mathbb{S}^{2}} f(\hat{\mathbf{z}} \cdot \hat{\mathbf{x}}) \operatorname{Im}\left(\mathrm{Y}_{n}^{m}(\hat{\mathbf{x}})\right) \mathrm{d} S(\hat{\mathbf{x}}) \\
& =\sum_{k=-n}^{n} \int_{\mathbb{S}^{2}} f\left(\hat{\mathbf{z}}^{\prime} \cdot \hat{\mathbf{x}}\right)\left[\operatorname{Re}\left(a_{k}\right) \operatorname{Im}\left(\mathrm{Y}_{n}^{k}(\hat{\mathbf{x}})\right)+\operatorname{Im}\left(a_{k}\right) \operatorname{Re}\left(\mathrm{Y}_{n}^{k}(\hat{\mathbf{x}})\right)\right] \mathrm{d} S(\hat{\mathbf{x}}) \\
& =\sum_{k=-n}^{n} \sqrt{\frac{(2 n+1)(n-|k|) !}{4 \pi(n+|k| !)}} \int_{0}^{\pi} \int_{0}^{2 \pi} f(\cos \theta) P_{n}^{|k|}(\cos \theta)\left[\operatorname{Re}\left(a_{k}\right) \sin (k \phi)+\operatorname{Im}\left(a_{k}\right) \cos (k \phi)\right] \mathrm{d} \phi \sin \theta \mathrm{d} \theta \\
& =\operatorname{Im}\left(a_{0}\right) \sqrt{\frac{2 n+1}{4 \pi}}\left(2 \pi \int_{0}^{\pi} f(\cos \theta) P_{n}(\cos \theta) \sin \theta \mathrm{d} \theta\right) .
\end{aligned}
$$

By substituting $\hat{\mathbf{x}}=\hat{\mathbf{z}}^{\prime}$ in $(97)$ and from $\mathrm{Y}_{n}^{k}\left(\hat{\mathbf{z}}^{\prime}\right)=0$ for $k \neq 0$,

$$
\mathrm{Y}_{n}^{m}(\hat{\mathbf{z}})=\mathrm{Y}_{n}^{m}\left(A \hat{\mathbf{z}}^{\prime}\right)=\sum_{k=-n}^{n} a_{k} \mathrm{Y}_{n}^{k}\left(\hat{\mathbf{z}}^{\prime}\right)=a_{0} \sqrt{\frac{2 n+1}{4 \pi}} P_{n}(1)=a_{0} \sqrt{\frac{2 n+1}{4 \pi}},
$$

so that

$$
\operatorname{Re}\left(\mathrm{Y}_{n}^{m}(\hat{\mathbf{z}})\right)=\operatorname{Re}\left(a_{0}\right) \sqrt{\frac{2 n+1}{4 \pi}} \text { and } \operatorname{Im}\left(\mathrm{Y}_{n}^{m}(\hat{\mathbf{z}})\right)=\operatorname{Im}\left(a_{0}\right) \sqrt{\frac{2 n+1}{4 \pi}} .
$$

Finally, (99), (100), and (102) imply (62) which complets the proof.

\section{References}

[1] S. Amstutz and N. Dominguez, "Topological sensitivity analysis in the context of ultrasonic nondestructive testing," Eng. Anal. Bound. Elem., vol. 32, pp. 936-947, 2008.

[2] T. Henriksson, M. Lambert, and D. Lesselier, "Non-iterative MUSIC-type algorithm for eddy-current nondestructive evaluation of metal plates," in Electromagnetic Nondestructive Evaluation (XIV), ser. Studies in Applied Electromagnetics and Mechanics, vol. 35, 2011, pp. 22-29.

[3] S. Verma, S. Bhadauria, and S. Akhtar, "Review of nondestructive testing methods for condition monitoring of concrete structures," J. Const. Eng, 2013.

[4] M. Haynes, J. Stang, and M. Moghaddam, "Microwave breast imaging system prototype with integrated numerical characterization," J. Biomed. Imag., vol. 2012, pp. 1-18, 2012.

[5] N. Irishina, M. Moscoso, and O. Dorn, "Microwave imaging for early breast cancer detection using a shape-based strategy," IEEE Trans. Biomed. Eng., vol. 56, pp. 1143-1153, 2009.

[6] B. Scholz, "Towards virtual electrical breast biopsy: space frequency MUSIC for trans-admittance data," IEEE Trans. Med. Imag., vol. 21, pp. 588-595, 2002.

[7] W. J. Baranoski, "Through-wall imaging: Historical perspective and future directions," J. Franklin Inst., vol. 345, pp. 556-569, 2008.

[8] D. Kim, B. Kim, and S. Nam, "A dual-band through-the-wall imaging radar receiver using a reconfigurable high-pass filter," J. Electromagn. Eng. Sci., vol. 16, pp. 164-168, 2016.

[9] F. Soldovieri and R. Solimene, "Through-wall imaging via a linear inverse scattering algorithm," IEEE Geoscience and Remote Sensing Letters, vol. 4, no. 4, pp. 513-517, 2007.

[10] H. Ammari, E. Iakovleva, and D. Lesselier, "A MUSIC algorithm for locating small inclusions buried in a half-space from the scattering amplitude at a fixed frequency," Multiscale Model. Simul., vol. 3, no. 3, pp. 597-628, 2006.

[11] R. Kress, "Inverse elastic scattering from a crack," Inverse Prob., vol. 12, pp. 667-684, 1996. 
[12] W.-K. Park, "Multi-frequency subspace migration for imaging of perfectly conducting, arc-like cracks in full- and limited-view inverse scattering problems," J. Comput. Phys., vol. 283, pp. 52-80, 2015.

[13] H. Ammari, J. Garnier, H. Kang, W.-K. Park, and K. Sølna, "Imaging schemes for perfectly conducting cracks," SIAM J. Appl. Math., vol. 71, pp. 68-91, 2011.

[14] M. Cetin, I. Stojanovic, O. Onhon, K. Varshney, S. Samadi, W. C. Karl, and A. S. Willsky, "Sparsitydriven synthetic aperture radar imaging: Reconstruction, autofocusing, moving targets, and compressed sensing," IEEE Signal Processing Magazine, vol. 31, no. 4, pp. 27-40, 2014.

[15] L. Zhang, M. Xing, C.-W. Qiu, J. Li, J. Sheng, Y. Li, and Z. Bao, "Resolution enhancement for inversed synthetic aperture radar imaging under low snr via improved compressive sensing," IEEE Transactions on Geoscience and Remote Sensing, vol. 48, no. 10, pp. 3824-3838, 2010.

[16] T. Zhang and X.-G. Xia, "Ofdm synthetic aperture radar imaging with sufficient cyclic prefix," IEEE Transactions on Geoscience and Remote Sensing, vol. 53, no. 1, pp. 394-404, 2015.

[17] I. Catapano, F. Soldovieri, and L. Crocco, "2D GPR imaging via linear sampling method: A performance assessment tool," in 6th International Workshop on Advanced Ground Penetrating Radar (IWAGPR). IEEE, 2011, pp. 1-4.

[18] X. Liu, M. Serhir, and M. Lambert, "Detectability of underground electrical cables junction with a ground penetrating radar: electromagnetic simulation and experimental measurements," Constr. Build. Mater., vol. 158, pp. 1099-1110, 2018.

[19] X. Liu, M. Serhir, A. Kameni, M. Lambert, and L. Pichon, "Ground penetrating radar data imaging via Kirchhoff migration method," in International Applied Computational Electromagnetics Society Symposium (ACES). IEEE, 2017, pp. 1-2.

[20] F. Soldovieri and R. Solimene, "Ground penetrating radar subsurface imaging of buried objects," in Radar Technology, G. Kouemou, Ed. Rijeka: IntechOpen, 2010, ch. 6.

[21] K. Ito, B. Jin, and J. Zou, "A direct sampling method to an inverse medium scattering problem," Inverse Prob., vol. 28, no. 2, p. 025003, 2012.

[22] J. Li and Z. Zou, "A direct sampling method for inverse scattering using far-field data," Inverse Probl. Imag., vol. 7, no. 3, pp. 757-775, 2013.

[23] W.-K. Park, "Direct sampling method for anomaly imaging from scattering parameter," Appl. Math. Lett., vol. 81, pp. $63-71,2018$.

[24] S. Kang, M. Lambert, and W.-K. Park, "Multi-frequency direct sampling method in inverse scattering problem," J. Phys: Conf. Ser., vol. 904, no. 1, p. 012018, 2017.

[25] W.-K. Park, "Direct sampling method for retrieving small perfectly conducting cracks," J. Comput. Phys., vol. 373, pp. $648-661,2018$.

[26] X. Liu, "A novel sampling method for multiple multiscale targets from scattering amplitudes at a fixed frequency," Inverse Problems, vol. 33, no. 8, p. 085011, jul 2017. [Online]. Available: https://doi.org/10.1088\%2F1361-6420\%2Faa777d

[27] R. Potthast, "A study on orthogonality sampling," Inverse Problems, vol. 26, no. 7, p. 074015, jul 2010. [Online]. Available: https://doi.org/10.1088\%2F0266-5611\%2F26\%2F7\%2F074015

[28] M. N. Akıncı, M. Çayören, and Akduman, "Near-field orthogonality sampling method for microwave imaging: Theory and experimental verification," IEEE Transactions on Microwave Theory and Techniques, vol. 64, no. 8, pp. 2489-2501, Aug 2016.

[29] M. N. Akınc1, "Improving near-field orthogonality sampling method for qualitative microwave imaging," IEEE Transactions on Antennas and Propagation, vol. 66, no. 10, pp. 5475-5484, Oct 2018.

[30] S. Kang, M. Lambert, and W.-K. Park, "Direct sampling method for imaging small dielectric inhomogeneities: analysis and improvement," Inverse Prob., vol. 34, no. 9, p. 095005 (18pp), 2018. 
[31] S. Kang, M. Lambert, and W. Park, "Analysis and improvement of direct sampling method in the monostatic configuration," IEEE Geosci. Remote. S., pp. 1-5, 2019.

[32] K. Ito, B. Jin, and J. Zou, "A direct sampling method for inverse electromagnetic medium scattering," Inverse Prob., vol. 29, no. 9, p. 095018, 2013.

[33] X. Chen and Y. Zhong, "Music electromagnetic imaging with enhanced resolution for small inclusions," Inverse Problems, vol. 25, no. 1, p. 015008, 2008.

[34] H. Ammari, E. Iakovleva, D. Lesselier, and G. Perrusson, "MUSIC-type electromagnetic imaging of a collection of small three-dimensional inclusions,", SIAM J. Sci. Comput., vol. 29, no. 2, pp. 674-709, 2007.

[35] R. Griesmaier, "A general perturbation formula for electromagnetic fields in presence of low volume scatterers," ESAIM: Mathematical Modelling and Numerical Analysis, vol. 45, no. 6, pp. 1193-1218, 2011.

[36] H. Ammari and D. Volkov, "Correction of order three for the expansion of two dimensional electromagnetic fields perturbed by the presence of inhomogeneities of small diameter," Journal of Computational Physics, vol. 189, no. 2, pp. 371-389, 2003.

[37] M. Deserno, "How to generate equidistributed points on the surface of a sphere," P.-If Polymerforshung (Ed.), p. 99, 2004.

[38] F. S. Bazán, J. B. Francisco, K. H. Leem, and G. Pelekanos, "Using the linear sampling method and an improved maximum product criterion for the solution of the electromagnetic inverse medium problem," Journal of Computational and Applied Mathematics, vol. 273, pp. 61-75, 2015.

[39] P. Jaccard, "The distribution of the flora in the alpine zone," New Phytol., vol. 11, no. 2, pp. 37-50, 1912.

[40] D. Colton and R. Kress, Inverse Acoustic and Electromagnetic Scattering Theory, 2nd ed. Springer, 1998.

[41] A. Kirsch and F. Hettlich, "The mathematical theory of maxwell's equations," Lecture notes, 2009.

[42] J. M. Geffrin and P. Sabouroux, "Continuing with the fresnel database: experimental setup and improvements in 3d scattering measurements," Inverse Problems, vol. 25, no. 2, p. 024001, 2009.

[43] J.-M. Geffrin, P. C. Chaumet, C. Eyraud, K. Belkebir, and P. Sabouroux, "Electromagnetic threedimensional reconstruction of targets from free space experimental data," Applied Physics Letters, vol. 92, no. 19, p. 194103, 2008. 